THE EXPECTED NUMBER OF NASH EQUILIBRIA

OF A NORMAL FORM GAME

by

Andrew McLennan

Discussion Paper No. 306, March 1999

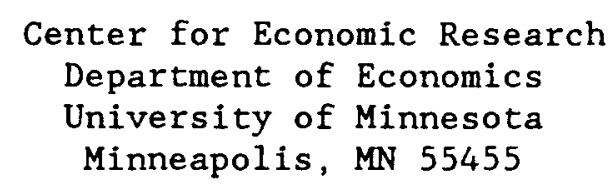




\title{
The Expected Number of Nash Equilibria of a Normal Form Game*
}

\author{
by \\ Andrew McLennan \\ Department of Economics \\ University of Minnesota \\ 271 19th Avenue South \\ Minneapolis, MN 55455 \\ mclennandatlas.socsci.umn.edu \\ http://www. econ.umn.edu/ mclennan
}

March, 1999

\footnotetext{
* This research was supported in part by National Science Foundation grant SBR-9308862 to the University of Minnesota. Software used to perform calculations reported herein used source code developed by the Gambit Project. I have benefited from numerous discussions with Richard McKelvey and Maurice Rojas.
} 


\begin{abstract}
Fix finite pure strategy sets $S_{1}, \ldots, S_{n}$, and let $S=S_{1} \times \ldots \times S_{n}$. In our model of a random game the agents' payoffs are statistically independent, with each agent's payoff uniformly distributed on the unit sphere in the dual of $\mathbb{R}^{S}$. For given nonempty $T_{1} \subset S_{1}, \ldots, T_{n} \subset S_{n}$ we give a computationally implementable formula for the mean number of Nash equilibria in which each agent $i$ 's mixed strategy has support $T_{i}$. The formula is the product of two expressions, where the first is the expected number of totally mixed equilibria for the truncated game obtained by eliminating pure strategies outside the sets $T_{i}$, and the second may be construed as the "probability" that such an equilibrium remains an equilibrium when the strategies in the sets $S_{i} \backslash T_{i}$ become available. The expected number of totally mixed equilibria for the truncated game is shown to be at least as large as $2^{-3 p / 2}$ times the square root of the maximal number of totally mixed equlibria (for generic payoffs) where $p=p_{1}+\ldots+p_{n}$ is the sum of the dimensions $p_{i}=\left|T_{i}\right|-1$ of the simplices of mixed strategies with support $T_{i}$. From this it follows that, in certain senses, the mean number of totally mixed equilibria grows exponentially as the size of the game increases. The expected number of Nash equilibria of all sorts is obtained by summing over supports. Numerical results which suggest certain monotonicity conjectures are presented. The distribution of equilibrium mixed strategy vectors is characterized. Journal of Economic Literature Classification Number C72.
\end{abstract}




\section{The Expected Number of Nash Equilibria of a Normal Form Game}

\section{Introduction}

The applicability of Nash equilibrium, and its variants and refinements, is limited in many ways by the complexity of the concept. If equilibrium is reached through a process of introspection, then it is necessary for each agent to compute at least her strategy in the equilibrium that is chosen, and possibly much more, depending on the principles that are thought to guide equilibrium selection. In a broader range of applications equilibrium is regarded as a stable pattern of social behavior that might result from evolution or learning. Convergence to equilibrium can occur without complicated calculations on the part of individuals, but although the burden of computational complexity is displaced to the level of the strategic adjustment process, it is still limiting.

This paper contributes to the quantification of these limitations using the methods and outlook of theoretical computer science. Specifically, we propose a model of a random normal form game for given (finite nonempty) sets of players and pure strategies; this model is shown to be canonical in a certain sense. We give a formula for the mean number of Nash equilibria that have a particular support ${ }^{(1)}$. The formula involves an integral that may be evaluated numerically without difficulty. We give a theoretical lower bound for this integral in terms of combinatorically defined quantities. This bound implies that, in a variety of senses, the mean number of equilibria grows exponentially with the size of the game.

Our work has the following features and consequences:

(A) The mean of the sum of a finite collection of random variables (no matter how correlated) is the sum of the means, so one may derive the expected number of Nash equilibria of all sorts from our formula by simple summation.

(B) The formula is the product of two factors, one of which is the expected number of totally mixed ${ }^{(2)}$ Nash equilibria for the truncated game obtained by eliminating pure strategies outside the support, while the second may be interpreted as the "probability" that a totally mixed equilibrium of the truncated game remains a Nash equilibrium when the strategies outside the support are available.

(C) The analysis depends on the application of methods from the mathematical literature on what are known as "sparse" systems of polynomial equations, and from a literature

- (cf. Edelman and Kostlan (1995)) that computes mean numbers of roots for a variety of polynomial systems. A companion paper (McLennan (1998b)) explores the key calculation as it would most naturally be posed in these literatures. In particular, we will apply a formula developed there that generalizes previous results by Kostlan (1993), Shub and Smale (1993), and Rojas (1996).

(D) The concept of Nash equilibrium for the given support, applied to the given distribution over games, induces a measure on the space of mixed strategy vectors, and

(1) The support of a Nash equilibrium specifies the pure strategies that are assigned positive probability.

(2) A Nash equilibrium is totally mixed if it assigns positive probability to all pure strategies. 
the mean given by our formula is the total mass of this measure. In fact we are able to characterize this measure completely. The measure is the product of measures on the agents' simplices of mixed strategies. Theorem 3 gives a closed form formula for the density of these factor measures. These measures are shown to be highly concentrated near the barycenters.

(E) Numerical computation of the mean number of equilibria is simple to program, and feasible for fairly large games. (The time consuming step is Monte Carlo approximation of the mean absolute value of a random square matrix with as many rows and columns as the dimension of the space of mixed strategies, which is the total number of pure strategies less the number of agents.) We present various computational results which, in addition to their intrinsic interest, suggest various monotonicity conjectures concerning the relationship between the numbers of pure strategies for the agents and the mean number of equilibria.

The remainder of this introduction discusses various conceptual points related to this work. The next section introduces enough of the model and the methods to allow us to state the key result, and to discuss various implications. The remainder of the paper will be described at the end of that section.

Other sciences (e.g. chemistry) describe notions of equilibrium in terms of solutions to equations which might have more than one solution, but the possibility of multiple equilibria is of special interest in game theory due to the doubts that surround the Nash equilibrium concept and its variants. (E.g., Luce and Raiffa (1957, 104-6) and Kreps $(1990,28-36)$.) There is, of course, a very large literature describing how forces tending in the direction of equilibrium might operate. (E.g., Fudenberg and Levine (1998), Harsanyi and Selten (1988), Weibull (1995)). Such theories might depend on computing the entire set of Nash equilibria, in which case the complexity of the computation is naturally related to the number of solutions. More commonly, equilibrium is thought of as a rest point, and perhaps an attractor, of a dynamic adjustment process, in which case the number of equilibria gives a measure of the complexity of the terrain that must be traversed if the process is to arrive at some equilibrium. Experimental literature provides a strong motivation for thinking about equilibration carefully, since in practice it is far from always the case that equilibrium is, in any sense, achieved. We will not discuss these literatures in any detail, but merely note that with respect to almost all such theorizing, the plausibility of mechanisms that might lead to Nash equilibrium declines rapidly as the complexity of the game increases.

Our approach to thinking about the complexity of normal form game theory is closely related to the formal measures of computational complexity studied in computer science. In order to be theoretically interesting, measures of the time or memory requirements of an algorithm should be independent of the particular machine on which the algorithm is run. For this reason the most intensively studied measures of complexity are quite coarse. Perhaps the best known distinction in computer science is between algorithms whose running times grow at a rate that is bounded by a polynomial function of the size of the input, in contrast with those whose time or memory requirements are bounded below by exponential functions of the input size. An important method in such analysis is to derive lower bounds on asymptotic complexity by simply counting the number of steps 
required to print the output.

Worst case running times are much more extensively studied than mean running times, not because they are thought to be of greater interest, but rather because of greater tractability. In order to study mean running times one must first of all settle on a probability distribution on the space of problems of a certain "size," or satisfying a certain combinatoric description. Usually the distribution that is most tractable, or otherwise most natural mathematically, is the focus of greatest interest, but this distribution can be criticized as "unrealistic." (For a concrete instance consider that in rather few of the actual applications of sorting algorithms is it the case that all orderings of the inputs are equiprobable.) Mathematically, the choice of distribution in this paper is justified by the fact that it is the unique measure satisfying natural and powerful symmetry conditions, by the tractability of the analysis given here and in McLennan (1998b), and by the apparent lack of any plausible alternative. We make no claim concerning the "realism" of this distribution as a model of the probability distribution of games occurring "in nature." Indeed, a large fraction of the games of interest to social scientists are artifacts of law, custom, or other forms of human design. It is natural to expect that designers of games would often feel that a simple and unambiguous equilibrium analysis was essential, and our results might sensibly be viewed as information related to the severity of this constraint.

For worst case analysis it often suffices to compute the running time of a particular instance of the problem, or to extract a worst case bound by patching together worst case estimates for different steps of the procedure, with each subsidiary bound possibly derived from the consideration of some well chosen instance of the problem. In contrast, the study of mean running times requires the analyst to control the entire space of problem instances, and to integrate over this space. This has proved possible only in a rather small subset of the computational problems and algorithms for which worst case analysis has been tractable. (Smale (1983) is an application to the simplex algorithm for linear programming.) Fortuitously, we are able to apply the methods in the literature on the distribution of roots of random polynomials (cf. Edelman and Kostlan (1995)) to the problems presented by normal form games. For several reasons the corresponding questions for extensive form game theory seem less tractable.

\section{The Model and the Main Result}

We now describe the model and just enough of our methods to allow the formula for the mean number of equilibria to be stated. Briefly, we adopt the standard setting of normal form game theory: there are finitely many agents, each of whom has a nonempty finite set of pure strategies. Our model of a random game is given by the uniform distribution on the cartesian product of the unit spheres in the spaces of utilities for each agent. Our

goal is to compute the expected number of Nash equilibria, for a given support. From a technical point of view, the main novelty is that we place mixed strategies in the positive orthant of the unit sphere, instead of the unit simplex.

\subsection{The Given Data}

Fix a number $n \geq 1$ of agents, and fix finite, nonempty sets of pure strategies 
$S_{1}, \ldots, S_{n}$. This information determines a normal form. The set of pure strategy vectors is

$$
S:=S_{1} \times \ldots \times S_{n}
$$

Nash equilibria may be classified by the strategies that are assigned positive probability. Fix a support $\mathbf{T}=\left(T_{1}, \ldots, T_{n}\right)$, where, for each $i=1, \ldots, n$,

$$
\emptyset \neq T_{i}:=\left\{t_{i}^{0}, t_{i}^{1}, \ldots, t_{i}^{p_{i}}\right\} \subset S_{i} .
$$

Let

$$
T:=T_{1} \times \ldots \times T_{n} \subset S
$$

be the pure strategy vectors whose components are in the support.

\subsection{The Model of a Random Game}

For the given normal form a normal form game is obtained by specifying a utility for each agent, where the space of relevant utilities is $\left(\mathbb{R}^{S}\right)^{*}$, the dual of $\mathbb{R}^{S}$. We will write either $y(z)$ or $\langle y, z\rangle$ to denote the evaluation of a linear functional $y \in\left(\mathbb{R}^{S}\right)^{*}$ at $z \in \mathbb{R}^{S}$.

Endowing $\mathbb{R}^{S}$ and $\left(\mathbb{R}^{S}\right)^{*}$ with the standard inner products and associated norms, let

$$
M:=M_{1} \times \ldots \times M_{n},
$$

where each $M_{i}$ is the unit sphere $\left(\mathbb{R}^{S}\right)^{*}$. In general, whenever $X$ is a $C^{1}$ submanifold of a Euclidean space, $\operatorname{vol}_{X}(\cdot)$ (or simply $\operatorname{vol}(\cdot)$ if there is no ambiguity) denotes the measure corresponding to the notion of volume derived from the inner product of the ambient space. When $\operatorname{vol}(X)$ is finite, the uniform distribution on $X$ is the probability measure $\mathrm{U}_{X}:=\operatorname{vol}_{X}(\cdot) / \operatorname{vol}_{X}(X)$. The model of a random game studied here is given by $\mathrm{U}_{M}$.

For any vector of utilities $u=\left(u_{1}, \ldots, u_{n}\right)$ with $u_{i} \neq 0$ for each $i$, the set of Nash equilibria is the same as the set of Nash equilibria for the vector of normalized utilities $\left(u_{1} /\left\|u_{1}\right\|, \ldots, u_{n} /\left\|u_{n}\right\|\right) \in M$. For our purposes, therefore, any model of a random game, by which we mean a distribution on $\left(\left(\mathbb{R}^{S}\right)^{*}\right)^{n}$ that assigns no probability to the set of vectors in which some agent's utility is 0 , is equivalent to the distribution on $M$ induced by this normalization. An interesting model that is equivalent to $\mathbf{U}_{M}$ is that the payoffs of the various agents at the various pure strategy vectors are i.i.d. normal random variables.

\subsection{Mixed Strategies and Expected Payoffs}

Variants and generalizations of the usual notion of a mixed strategy for agent $i$ will be drawn from $\mathbb{R}^{S_{i}}$, whose elements are typically denoted by $\sigma, \sigma^{\prime}, \ldots$ Elements of the subspace $\mathbb{R}^{T_{i}}$ are typically denoted by $\tau_{i}, \tau_{i}^{\prime}, \ldots$ For $\sigma \in \mathbb{R}^{S_{1}} \times \ldots \times \mathbb{R}^{S_{n}}$ and $\sigma_{i}^{\prime} \in \mathbb{R}^{S_{i}}$ let

$$
\sigma \mid \sigma_{i}^{\prime}:=\left(\sigma_{1}, \ldots, \sigma_{i-1}, \sigma_{i}^{\prime}, \sigma_{i+1}, \ldots, \sigma_{n}\right) .
$$

We will not make a notational distinction between a pure strategy $s_{i} \in S_{i}$ and the corresponding unit vector in $\mathbb{R}^{S_{i}}$. 
To compute expected utilities we need to describe the probability distribution on pure strategy vectors induced by a vector of mixed strategies. This is a product measure, and it is computed by the multilinear function

$$
\Theta: \mathbb{R}^{S_{1}} \times \ldots \times \mathbb{R}^{S_{n}} \rightarrow \mathbb{R}^{S}
$$

with $s$-coordinate function $\Theta_{s}(\sigma):=\prod_{i=1}^{n} \sigma_{i}\left(s_{i}\right)$. For $\sigma \in \mathbb{R}^{S_{1}} \times \ldots \times \mathbb{R}^{S_{n}}$ and $u_{i} \in\left(\mathbb{R}^{S}\right)^{*}$ let $u_{i}(\sigma):=\left\langle u_{i}, \Theta(\sigma)\right\rangle$ be the generalization of the usual notion of expected utility.

\subsection{The Equilibrium Correspondences}

The following conditions characterize the notion that $\tau$ is a strict ${ }^{(3)}$ Nash equilibrium with support $\mathbf{T}$ for utility $u$.

(N1) $u_{i}\left(\tau \mid t_{i}\right)=u_{i}\left(\tau \mid t_{i}^{\prime}\right)$ for all $i$ and $t_{i}, t_{i}^{\prime} \in T_{i}$.

(N2) $u_{i}\left(\tau \mid t_{i}\right)>u\left(\tau \mid s_{i}\right)$ for all $i, t_{i} \in T_{i}$ and $s_{i} \in S_{i} \backslash T_{i}$.

(N3) $\tau_{i}$ is in the interior of the positive orthant of $\mathbb{R}^{T_{i}}$ for all $i$.

(N4) for all $i$ the sum of the components of $\tau_{i}$ is one.

Note that if one of (N1), (N2), or (N3) is satisfied by $u$ and $\tau$, then, for any positive scalars $\alpha_{1}, \ldots, \alpha_{n}$, it also holds for $u$ and $\left(\alpha_{1} \tau_{1}, \ldots, \alpha_{n} \tau_{n}\right)$. Mathematically it is most natural to think of one of the roots we are counting as a cartesian product of rays emanating from the origin in the various spaces $\mathbb{R}^{T_{i}}$. While (N4) is conceptually natural, of course, for our work here it is only one method of selecting a canonical point from such a product of rays. As we proceed into the analysis it will become abundantly clear that a different "normalization," the requirement that each $\tau_{i}$ lie in the unit sphere in $\mathbb{R}^{T_{i}}$, is much more fruitful mathematically. Let

$$
N:=N_{1} \times \ldots \times N_{n} \text { and } N^{++}:=N_{1}^{++} \times \ldots \times N_{n}^{++}
$$

where, for each $i, N_{i}$ is the unit sphere in $\mathbb{R}^{T_{i}}$ and $N_{i}^{++}$is the intersection of $N_{i}$ with the interior of the positive orthant.

In the analysis the most important analogue of the graph of the equilibrium correspondence will be

$$
V=\{(u, \tau) \in M \times N:(\mathrm{N} 1)\} .
$$

Let $\pi_{1}: V \rightarrow M$ and $\pi_{2}: V \rightarrow N$ be the restrictions to $V$ of the natural projections from $M \times N$. Let $\nu$ the measure on $N$ defined, for measurable $E \subset N$, by

$$
\nu(E)=\int_{M} \#\left(\pi_{1}^{-1}(u) \cap \pi_{2}^{-1}(E)\right) d \mathbf{U}_{M}(u) .
$$

In the analysis of partially mixed equilibrium we study the following open subset of $V$ :

$$
\tilde{V}=\{(u, \tau) \in V:(\mathrm{N} 2)\} .
$$

(3) The qualifier 'strict' refers to the fact that the inequalities in (N2) are required to hold strictly. Harsanyi (1973) demonstrates that on a generic set of utilities there are only strict equilibria, so it does not affect the expected number of equilibria if we consider only strict equilibria, as we shall in order to simplify the presentation. 
Let $\tilde{\nu}$ be the measure on $N$ defined, for measurable $E \subset N$, by

$$
\tilde{\nu}(E)=\int_{M} \#\left(\pi_{1}^{-1}(u) \cap \tilde{V} \cap \pi_{2}^{-1}(E)\right) d \mathbf{U}_{M}(u)
$$

Our ambition is to study the distribution of partially mixed equilibria induced by a random game, which means that we wish to characterize the measure on $\mathrm{N}^{++}$obtained by restricting $\tilde{\nu}$ to this set. In particular, $\tilde{\nu}\left(N^{++}\right)$is the mean number of partially mixed equilibria with support $\mathbf{T}$.

\subsection{Statement of the Main Result}

The results described below refer to the following objects. Let

$$
p:=p_{1}+\ldots+p_{n}
$$

be the sum of the dimensions of the agent's simplices (or sphere orthants) of mixed strategies. Effectively $p$ is the number of variables in the systems of polynomial equations we are studying. Let $q:\{1, \ldots, p\} \rightarrow\{1, \ldots, n\}$ be the function defined implicitly by the inequality

$$
p_{1}+\ldots+p_{q(k)-1}<k \leq p_{1}+\ldots+p_{q(k)} .
$$

Let $\delta(\mathbf{p})$ be the $p \times n$ matrix with entries

$$
\delta_{k i}(\mathbf{p})= \begin{cases}0 & \text { if } q(k)=i \\ 1 & \text { otherwise }\end{cases}
$$

Let $\bar{Z}_{\mathbf{p}}$ be a $p \times p$ random matrix whose rows are indexed by the integers $k=1, \ldots, p$, whose columns are indexed by the pairs $i j$ for $i=1, \ldots, n$ and $j=1, \ldots, p_{i}$, and whose entries $\tilde{z}_{k}^{i j}$ are independently distributed normal random variables with mean zero and variance $\delta_{k i}(\mathbf{p})$. Let $\Gamma:(0, \infty) \rightarrow(0, \infty)$ be Euler's function: $\Gamma(s) \equiv \int_{0}^{\infty} \exp (-x) x^{s-1} d x$.

Although there are many related ideas, the mathematical work breaks down into the two large parts expressed in the following two propositions, which are proved in Sections 6 and 7 respectively.

Proposition 2.1: For all measurable $E \subset N$,

$$
\nu(E)=\mathbf{U}_{N}(E) \cdot 2^{n-\frac{p}{2}} \cdot\left(\prod_{i=1}^{n} \frac{\Gamma\left(\frac{1}{2}\right)}{\Gamma\left(\frac{p_{i}+1}{2}\right)}\right) \cdot \mathbf{E}\left(\left|\operatorname{det} \tilde{Z}_{\mathbf{p}}\right|\right)
$$

For integers $a, b \geq 0$ define $r(a, b)$ to be the probability of the event $\epsilon_{0} / \sqrt{a+1}>$ $\max \left\{\epsilon_{1}, \ldots \epsilon_{b}\right\}$ when $\epsilon_{0}, \epsilon_{1}, \ldots, \epsilon_{b}$ are i.i.d. normal random variables with mean zero.

Proposition 2.2: For all measurable $E \subset N$ with $\nu(E)>0$,

$$
\frac{\tilde{\nu}(E)}{\nu(E)}=\prod_{i=1}^{n} r\left(p_{i},\left|S_{i} \backslash T_{i}\right|\right)
$$


The following combines the last two results into a single assertion.

Theorem 1: For all measurable $E \subset N$,

$$
\tilde{\nu}(E)=\mathbf{U}_{N}(E) \cdot\left(\prod_{i=1}^{n} r\left(p_{i},\left|S_{i} \backslash T_{i}\right|\right)\right) \cdot 2^{n-\frac{p}{2}} \cdot\left(\prod_{i=1}^{n} \frac{\Gamma\left(\frac{1}{2}\right)}{\Gamma\left(\frac{p_{i}+1}{2}\right)}\right) \cdot \mathbf{E}\left(\left|\operatorname{det} \tilde{Z}_{\mathbf{p}}\right|\right) .
$$

The following, which is perhaps the statement of greatest conceptual interest, follows from the fact that $\operatorname{vol}\left(N^{++}\right) / \operatorname{vol}(N)=2^{-(p+n)}$.

\section{Corollary:}

$$
\tilde{\nu}\left(N^{++}\right)=\left(\prod_{i=1}^{n} r\left(p_{i},\left|S_{i} \backslash T_{i}\right|\right)\right) \cdot 2^{-\frac{3 p}{2}} \cdot\left(\prod_{i=1}^{n} \frac{\Gamma\left(\frac{1}{2}\right)}{\Gamma\left(\frac{p_{i}+1}{2}\right)}\right) \cdot \mathbf{E}\left(\left|\operatorname{det} \tilde{Z}_{\mathbf{p}}\right|\right) .
$$

It is now possible to comment more fully on several of the points raised at the beginning of the paper, and on other matters.

Remark 1: The expectation of a sum of random variables, which may be correlated in any way whatsoever, is the sum of their expectations, so the mean number of Nash equilibria of all sorts, and the mean number of equilibria with support lying in any subset of the set of possible supports, are obtained by simple addition of the quantities considered in the results above.

Remark 2: Harsanyi (1973) shows that, for almost all $u$, all Nash equilibria are regular ${ }^{(4)}$, and consequently survive almost all the criteria for eliminating some Nash equilibria that are developed in the refinements literature. (Cf. van Damme (1987).) Perhaps the main exception is the refinement that excludes equilibria with index -1. (Generically each equilibrium has an index that may be either 1 or -1 , and the sum of these over all equilibria is 1; cf. Gul, Pearce, and Stacchetti (1993).) It is not apparent how one might use the methods developed here to compute the expected number of equilibria with index 1 on a support-by-support basis, but since, generically, the indices of the equilibria sum to one, the mean number of Nash equilibria with index -1 will be one less than the mean number of equilibria of index 1 , so both of these means are known once we know the mean number of Nash equilibria of both sorts.

Remark 3: The natural interpretation, that Proposition 2.1 characterizes the distribution of totally mixed Nash equilibria of the truncated game obtained by eliminating strategies in the sets $S_{i} \backslash T_{i}$, while Proposition 2.2 gives the probability that such an equilibrium is a partially mixed equilibrium of the original game, is correct in the following technical sense. The distribution on games for the strategy sets $T_{1}, \ldots, T_{n}$ obtained from the given

(4) A Nash equilibrium is regular if it is strict and is a regular point of the function taking $\tau \in N$ to the vector with components $u_{i}\left(\tau \mid t_{i}^{j}\right)-u_{i}\left(\tau \mid t_{i}^{0}\right)\left(i=1, \ldots, n, j=1, \ldots, p_{i}\right)$. 
distribution by taking the restrictions of the (random) linear functionals $u_{i} \in\left(\mathbb{R}^{S}\right)^{*}$ to $\mathbb{R}^{T} \subset \mathbb{R}^{S}$ is equivalent, in the sense discussed in $\S 2.2$, to the given distribution for our model of a random game with pure strategy sets $T_{1}, \ldots, T_{n}$.

Remark 4: Pure strategy equilibria correspond to the case $p_{1}=\ldots=p_{n}=0$, so that each $T_{i}$ is a singleton. Considering first the possibility that the sets $S_{i} \backslash T_{i}$ are all empty, we see that the correctness of (2) depends on the natural but conventional agreement that the determinant of a $0 \times 0$ matrix is unity. Clearly $r(0, b)=1 /(b+1)$ for any $b$, so for nonempty $S_{i} \backslash T_{i}$ the expected number of equilibria is

$$
\prod_{i=1}^{n} \frac{1}{\left|S_{i}\right|}
$$

Summing over all vectors of pure strategies, the expected number of pure Nash equilibria is seen to be one. These results hold somewhat more generally, namely whenever the payoffs for the various agents at the various pure strategy vectors are i.i.d. random variables whose common distribution has no mass points, since $\left|S_{i}\right|^{-1}$ is then the probability that agent $i$ 's component of a pure strategy vector is a best response to that vector. These results are due to Dresher (1970) and have been extended by Powers (1990) and Stanford (1993) to the point where the distribution of the number of pure Nash equilibria is quite well understood.

Remark 5: McKelvey and McLennan (1997) show that " $p_{i} \leq \sum_{h \neq i} p_{h}$ for all $i "$ is a necessary and sufficient condition for there to exist open sets of payoffs for which there are totally mixed Nash equilibria. Here this corresponds to the fact that (with probability one) $\tilde{Z}_{\mathbf{p}}$ has a $p_{i} \times p_{i}$ submatrix of zeros, so that $\operatorname{det}\left(\tilde{Z}_{\mathbf{p}}\right)=0$ when $p_{i}>p / 2$.

Remark 6: We now consider the case of $n=2$ agents. From the last remark it follows that the expected number of equilibria is 0 unless $p_{1}=p_{2}$. When $p_{1}=p_{2}$, for generic utilities the equations expressing agent 1 's indifference between all strategies in $T_{1}$ will determine a 1-dimensional linear subspace of $\mathbb{I}^{T_{2}}$, and similarly with agents reversed. By virtue of the symmetry conditions developed in the next two sections, the "probability" that each subspace will intersect the positive orthant is $2^{-p}$, and we arrive at the conclusion that the expected number of Nash equilibria with support $\mathbf{T}$ is

$$
2^{-p} \cdot r\left(p_{1},\left|S_{1} \backslash T_{1}\right|\right) \cdot r\left(p_{2},\left|S_{2} \backslash T_{2}\right|\right)
$$

Various analytic facts concerning the function $r$ are presented in Appendix A.

The remainder of the paper has the following organization. The next three sections discuss the results of computations of the mean number of totally mixed equilibria and the mean number of Nash equilibria of all sorts. Two agent games are discussed in Section 3 . Section 4 presents numerical findings concerning the mean number of Nash equilibria for games with more than two agents, with special attention to conjectures suggested by the calculations. Section 5 describes related mathematical work that implies that $2^{-p}$ times the square root of the maximal number of totally mixed equilibria is a lower bound on the expected number of totally mixed equilibria. Sections 6 and 7 prove Theorem 1 by 
establishing Propositions 2.1 and 2.2 respectively. Section 8 analyzes the distribution of equilibrium vectors of mixed strategies in the usual cartesian product of simplices that is implied by Theorem 1 .

Section 5 through 7 must be read in sequence. Otherwise the various sections and Appendix A are logically independent, and may be read with understanding in any order.

\section{Two Person Games}

The further study of the mean number (3) of equilibria for two agent games is a matter of studying the function $r$. Appendix A collects a variety of results concerning this function, including two lower bounds, but while these have some interest, they are not powerful enough to give information concerning the mean number of Nash equilibria of all sorts, which is obtained by summing (3) over the supports in which the two agents have the same number of pure strategies:

$$
\sum_{q=0}^{\min \left\{\left|S_{1}\right|,\left|S_{2}\right|\right\}-1} 2^{-2 q}\left(\begin{array}{c}
\left|S_{1}\right| \\
q+1
\end{array}\right)\left(\begin{array}{c}
\left|S_{2}\right| \\
q+1
\end{array}\right) \cdot r\left(q,\left|S_{1}\right|-q-1\right) \cdot r\left(q,\left|S_{2}\right|-q-1\right) .
$$

For example, Lemma A.6 shows that

$$
r(a, b) \geq\left(\frac{a+b+1}{a+1}\right)^{-1},
$$

from which we may compute that the mean number of Nash equilibria of all sorts is not less than

$$
\begin{gathered}
\sum_{q=0}^{\min \left\{\left|S_{1}\right|,\left|S_{2}\right|\right\}-1} 2^{-2 q}\left(\begin{array}{c}
\left|S_{1}\right| \\
q+1
\end{array}\right)\left(\begin{array}{c}
\left|S_{2}\right| \\
q+1
\end{array}\right)\left(\begin{array}{c}
\left|S_{1}\right| \\
q+1
\end{array}\right)^{-1}\left(\begin{array}{c}
\left|S_{2}\right| \\
q+1
\end{array}\right)^{-1} \\
=\sum_{q=0}^{\min \left\{\left|S_{1}\right|,\left|S_{2}\right|\right\}-1} 2^{-2 q}=\frac{4}{3}\left(1-2^{-2 \min \left\{\left|S_{1}\right| \cdot\left|S_{2}\right|\right\}}\right) .
\end{gathered}
$$

This "improves" the lower bound of one given by the Nash existence theorem, but just barely.

Since theoretical methods are so unproductive, computation provides the best sense of how the expected number of equilibria grows with the numbers of pure strategies. Let $\nu\left(\left|S_{1}\right|,\left|S_{2}\right|\right)$ be the expected number of Nash equilibria of all sorts, and let $\nu_{j}\left(\left|S_{1}\right|,\left|S_{2}\right|\right)$ $\left(1 \leq j \leq \min \left\{\left|S_{1}\right|,\left|S_{2}\right|\right\}\right)$ be the expected number of equilibria in which both players mix over $j$ pure strategies. Tables $1,2,3$, and 4 present the values of $\nu_{j}$ for $j=2,3,4,5$, respectively, and Table 5 presents the values of $\nu$. We will describe certain regularities exhibited by the total number of equilibria, remarking as we go along on how the data shown in Tables 1-4 does and does not share the properties of its aggregation in Table 5.

The most obvious feature of Table 5 , and perhaps the most important, is the observation that the expected number of Nash equilibria is increasing in $\left|S_{1}\right|$ and $\left|S_{2}\right|$. Monotonicity appears to also be a property of the functions $\nu_{j}$. There is a striking contrast between the 
simplicity and fundamental character of this property of the data, on the one hand, and the difficulty of extracting enough information about $r$ to be able to prove that this is true in general.

Examining second differences, in all cases examined to date it is the case that

$$
\left[\nu\left(m_{1}+2, m_{2}\right)-\nu\left(m_{1}+1, m_{2}\right)\right]-\left[\nu\left(m_{1}+1, m_{2}\right)-\nu\left(m_{1}, m_{2}\right)\right]<0
$$

(Here the whole is simpler than the parts of which it is the sum: for fixed $m_{2}, \nu_{j}\left(\cdot, m_{2}\right)$ may be an S-shaped function, as, for instance, in the $m_{2}=4$ column of Table 5.) That is, if the number of strategies for one player is held fixed while the number for the other increases, the expected number of equilibria increases at a decreasing rate. Table 5 strongly suggests that, for any $m_{2}, \nu\left(m_{1}+1, m_{2}\right)-\nu\left(m_{1}, m_{2}\right) \rightarrow 0$ as $m_{1} \rightarrow \infty$. The data seems consistent with the conjecture that $\lim _{m_{1} \rightarrow \infty} \nu\left(m_{1}, m_{2}\right)<\infty$ for any $m_{2}$, but the evidence is less compelling.

On the other hand Table 5 suggests that the second diagonal difference

$$
\left[\nu\left(m_{1}+2, m_{2}+2\right)-\nu\left(m_{1}+1, m_{2}+1\right)\right]-\left[\nu\left(m_{1}+1, m_{2}+1\right)-\nu\left(m_{1}, m_{2}\right)\right]
$$

is always positive. (The analogous property of $\nu_{j}$ fails when $j=2$.) That is, as one increases the numbers of strategies for both agents, the expected number of equilibria increases at an increasing rate. While this may be, a more subjective reaction to Table 5 is that the rate of acceleration is mild over a fairly broad range.

For the 'mixed' second difference, all experience to date is consistent with the conjecture that

$$
\left[\nu_{j}\left(m_{1}+1, m_{2}+1\right)-\nu_{j}\left(m_{1}+1, m_{2}\right)\right]-\left[\nu_{j}\left(m_{1}, m_{2}+1\right)-\nu_{j}\left(m_{1}, m_{2}\right)\right] \geq 0 .
$$

so that $\nu$ has this property by virtue of being the sum of the $\nu_{j}$. That is, the rate at which the number of equilibria increases with $m_{2}$ is an increasing function of $m_{1}$.

All computations to date are consistent with the conjecture that, for all $j$, the "diagonal difference" $\nu_{j}\left(m_{1}+1, m_{2}\right)-\nu_{j}\left(m_{1}, m_{2}+1\right)$ is positive if and only if $m_{1}<m_{2}-1$. This means that for any total number of pure strategies for the two agents, the expected nunber of equilibria will be greater if the strategies are divided more equally betueen the two agents. 


\begin{tabular}{|c|c|c|c|c|c|c|c|}
\hline \multicolumn{8}{|c|}{ Expected Numbers of Equilibria With Two Element Supports } \\
\hline $\begin{array}{c}\text { Numbers of } \\
\text { Strategies }\end{array}$ & $\mathbf{2}$ & $\mathbf{3}$ & $\mathbf{4}$ & $\mathbf{5}$ & $\mathbf{6}$ & $\mathbf{7}$ & $\mathbf{8}$ \\
\hline $\mathbf{2}$ & 0.250 & 0.375 & 0.456 & 0.515 & 0.562 & 0.599 & 0.631 \\
$\mathbf{3}$ & 0.375 & 0.563 & 0.684 & 0.773 & 0.842 & 0.899 & 0.946 \\
$\mathbf{4}$ & 0.456 & 0.684 & 0.832 & 0.940 & 1.024 & 1.093 & 1.151 \\
$\mathbf{5}$ & 0.515 & 0.773 & 0.940 & 1.062 & 1.157 & 1.235 & 1.300 \\
$\mathbf{6}$ & 0.562 & 0.842 & 1.024 & 1.157 & 1.261 & 1.346 & 1.417 \\
$\mathbf{7}$ & 0.599 & 0.899 & 1.093 & 1.235 & 1.346 & 1.436 & 1.512 \\
$\mathbf{8}$ & 0.631 & 0.946 & 1.151 & 1.300 & 1.417 & 1.512 & 1.592 \\
$\mathbf{9}$ & 0.658 & 0.987 & 1.201 & 1.356 & 1.478 & 1.577 & 1.660 \\
$\mathbf{1 0}$ & 0.682 & 1.023 & 1.244 & 1.405 & 1.531 & 1.634 & 1.720 \\
$\mathbf{1 1}$ & 0.703 & 1.054 & 1.283 & 1.449 & 1.579 & 1.685 & 1.774 \\
$\mathbf{1 2}$ & 0.722 & 1.083 & 1.317 & 1.488 & 1.622 & 1.730 & 1.822 \\
$\mathbf{1 3}$ & 0.739 & 1.109 & 1.349 & 1.524 & 1.660 & 1.771 & 1.865 \\
$\mathbf{1 4}$ & 0.755 & 1.132 & 1.377 & 1.556 & 1.695 & 1.809 & 1.905 \\
$\mathbf{1 5}$ & 0.769 & 1.154 & 1.403 & 1.586 & 1.728 & 1.844 & 1.941 \\
\hline
\end{tabular}

Table 1

\begin{tabular}{|c|c|c|c|c|c|c|c|}
\hline \multicolumn{6}{|c|}{ Expected Numbers of Equilibria With Three Element Supports } \\
\hline $\begin{array}{c}\text { Numbers of } \\
\text { Strategies }\end{array}$ & $\mathbf{2}$ & $\mathbf{3}$ & $\mathbf{4}$ & $\mathbf{5}$ & $\mathbf{6}$ & $\mathbf{7}$ & $\mathbf{8}$ \\
\hline $\mathbf{2}$ & & & & & & & \\
$\mathbf{3}$ & & 0.063 & 0.125 & 0.181 & 0.232 & 0.277 & 0.317 \\
$\mathbf{4}$ & 0.125 & 0.250 & 0.363 & 0.463 & 0.553 & 0.635 \\
$\mathbf{5}$ & & 0.181 & 0.363 & 0.526 & 0.672 & 0.803 & 0.921 \\
$\mathbf{6}$ & & 0.232 & 0.463 & 0.672 & 0.859 & 1.025 & 1.176 \\
$\mathbf{7}$ & & 0.277 & 0.553 & 0.803 & 1.025 & 1.225 & 1.405 \\
$\mathbf{8}$ & & 0.317 & 0.635 & 0.921 & 1.176 & 1.405 & 1.611 \\
$\mathbf{9}$ & & 0.354 & 0.709 & 1.028 & 1.313 & 1.568 & 1.799 \\
$\mathbf{1 0}$ & & 0.388 & 0.776 & 1.127 & 1.439 & 1.718 & 1.971 \\
$\mathbf{1 1}$ & 0.419 & 0.839 & 1.217 & 1.555 & 1.857 & 2.130 \\
$\mathbf{1 2}$ & & 0.448 & 0.897 & 1.302 & 1.662 & 1.985 & 2.277 \\
$\mathbf{1 3}$ & & 0.476 & 0.951 & 1.380 & 1.763 & 2.105 & 2.414 \\
$\mathbf{1 4}$ & & 0.501 & 1.002 & 1.454 & 1.857 & 2.217 & 2.543 \\
$\mathbf{1 5}$ & & 0.525 & 1.049 & 1.523 & 1.945 & 2.323 & 2.664 \\
\hline
\end{tabular}

Table 2 


\begin{tabular}{|c|c|c|c|c|c|c|c|}
\hline \multicolumn{6}{|c|}{ Expected Numbers of Equilibria With Four Element Supports } \\
\hline $\begin{array}{c}\text { Numbers of } \\
\text { Strategies }\end{array}$ & $\mathbf{2}$ & $\mathbf{3}$ & $\mathbf{4}$ & $\mathbf{5}$ & $\mathbf{6}$ & $\mathbf{7}$ & $\mathbf{8}$ \\
\hline $\mathbf{2}$ & & & & & & & \\
$\mathbf{3}$ & & & & & & \\
$\mathbf{4}$ & & 0.016 & 0.039 & 0.066 & 0.095 & 0.124 \\
$\mathbf{5}$ & & 0.039 & 0.098 & 0.165 & 0.237 & 0.309 \\
$\mathbf{6}$ & & 0.066 & 0.165 & 0.280 & 0.400 & 0.523 \\
$\mathbf{7}$ & & 0.095 & 0.237 & 0.400 & 0.573 & 0.749 \\
$\mathbf{8}$ & & 0.124 & 0.309 & 0.523 & 0.749 & 0.978 \\
$\mathbf{9}$ & & 0.152 & 0.381 & 0.645 & 0.923 & 1.206 \\
$\mathbf{1 0}$ & & 0.181 & 0.452 & 0.765 & 1.095 & 1.430 \\
$\mathbf{1 1}$ & & 0.208 & 0.521 & 0.882 & 1.263 & 1.649 \\
$\mathbf{1 2}$ & & 0.235 & 0.589 & 0.996 & 1.426 & 1.863 \\
$\mathbf{1 3}$ & & 0.262 & 0.654 & 1.107 & 1.585 & 2.070 \\
$\mathbf{1 4}$ & & 0.287 & 0.718 & 1.215 & 1.740 & 2.272 \\
$\mathbf{1 5}$ & & 0.312 & 0.780 & 1.320 & 1.890 & 2.468 \\
\hline
\end{tabular}

Table 3

\begin{tabular}{|c|c|c|c|c|c|c|c|}
\hline \multicolumn{6}{|c|}{ Expected Numbers of Equilibria With Five Element Supports } \\
\hline $\begin{array}{c}\text { Numbers of } \\
\text { Strategies }\end{array}$ & $\mathbf{2}$ & $\mathbf{3}$ & $\mathbf{4}$ & $\mathbf{5}$ & $\mathbf{6}$ & $\mathbf{7}$ & $\mathbf{8}$ \\
\hline $\mathbf{2}$ & & & & & & & \\
$\mathbf{3}$ & & & & & & \\
$\mathbf{4}$ & & & & & & \\
$\mathbf{5}$ & & & 0.004 & 0.012 & 0.023 & 0.036 \\
$\mathbf{6}$ & & & 0.012 & 0.035 & 0.068 & 0.108 \\
$\mathbf{7}$ & & & 0.023 & 0.068 & 0.132 & 0.210 \\
$\mathbf{8}$ & & & 0.036 & 0.108 & 0.210 & 0.333 \\
$\mathbf{9}$ & & & 0.051 & 0.154 & 0.298 & 0.474 \\
$\mathbf{1 0}$ & & & 0.068 & 0.204 & 0.394 & 0.627 \\
$\mathbf{1 1}$ & & & 0.085 & 0.256 & 0.496 & 0.789 \\
$\mathbf{1 2}$ & & & 0.104 & 0.311 & 0.602 & 0.958 \\
$\mathbf{1 3}$ & & & 0.123 & 0.368 & 0.712 & 1.132 \\
$\mathbf{1 4}$ & & & 0.142 & 0.425 & 0.823 & 1.309 \\
$\mathbf{1 5}$ & & & 0.161 & 0.483 & 0.936 & 1.489 \\
\hline
\end{tabular}

Table 4 


\begin{tabular}{|c|c|c|c|c|c|c|c|}
\hline \multicolumn{8}{|c|}{ Expected Numbers of Nash Equilibria of All Sorts } \\
\hline $\begin{array}{c}\text { Numbers of } \\
\text { Strategies }\end{array}$ & 2 & 3 & 4 & 5 & 6 & 7 & 8 \\
\hline 2 & 1.250 & 1.375 & 1.456 & 1.515 & 1.562 & 1.599 & 1.631 \\
\hline 3 & 1.375 & 1.625 & 1.809 & 1.954 & 2.074 & 2.175 & 2.264 \\
\hline 4 & 1.456 & 1.809 & 2.098 & 2.342 & 2.554 & 2.741 & 2.909 \\
\hline 5 & 1.515 & 1.954 & 2.342 & 2.690 & 3.007 & 3.297 & 3.566 \\
\hline 6 & 1.562 & 2.074 & 2.554 & 3.007 & 3.436 & 3.843 & 4.232 \\
\hline 7 & 1.599 & 2.175 & 2.741 & 3.297 & 3.843 & 4.378 & 4.902 \\
\hline 8 & 1.631 & 2.264 & 2.909 & 3.566 & 4.232 & 4.902 & 5.575 \\
\hline 9 & 1.658 & 2.341 & 3.062 & 3.817 & 4.603 & 5.415 & 6.248 \\
\hline 10 & 1.682 & 2.411 & 3.201 & 4.052 & 4.959 & 5.916 & 6.920 \\
\hline 11 & 1.703 & 2.474 & 3.330 & 4.273 & 5.300 & 6.408 & 7.590 \\
\hline 12 & 1.722 & 2.531 & 3.450 & 4.482 & 5.629 & 6.888 & 8.257 \\
\hline 13 & 1.739 & 2.584 & 3.561 & 4.680 & 5.946 & 7.359 & 8.920 \\
\hline 14 & 1.755 & 2.633 & 3.666 & 4.869 & 6.252 & 7.820 & 9.579 \\
\hline 15 & 1.769 & 2.679 & 3.765 & 5.050 & 6.548 & 8.273 & 10.233 \\
\hline 16 & 1.783 & 2.721 & 3.858 & 5.222 & 6.835 & 8.716 & 10.882 \\
\hline 17 & 1.795 & 2.761 & 3.947 & 5.388 & 7.113 & 9.151 & 11.525 \\
\hline 18 & 1.806 & 2.799 & 4.031 & 5.547 & 7.384 & 9.578 & 12.164 \\
\hline 19 & 1.817 & 2.834 & 4.112 & 5.700 & 7.647 & 9.997 & 12.797 \\
\hline 20 & 1.828 & 2.868 & 4.189 & 5.848 & 7.903 & 10.409 & 13.425 \\
\hline 21 & 1.837 & 2.900 & 4.262 & 5.991 & 8.152 & 10.814 & 14.048 \\
\hline 22 & 1.846 & 2.930 & 4.333 & 6.129 & 8.395 & 11.212 & 14.665 \\
\hline 23 & 1.855 & 2.959 & 4.401 & 6.263 & 8.632 & 11.604 & 15.277 \\
\hline 24 & 1.863 & 2.987 & 4.466 & 6.393 & 8.864 & 11.989 & 15.883 \\
\hline 25 & 1.871 & 3.014 & 4.530 & 6.519 & 9.091 & 12.368 & 16.485 \\
\hline 26 & 1.878 & 3.040 & 4.591 & 6.641 & 9.313 & 12.742 & 17.081 \\
\hline 27 & 1.885 & 3.064 & 4.650 & 6.760 & 9.530 & 13.110 & 17.672 \\
\hline 28 & 1.892 & 3.088 & 4.707 & 6.876 & 9.742 & 13.472 & 18.258 \\
\hline 29 & 1.899 & 3.111 & 4.762 & 6.989 & 9.950 & 13.830 & 18.839 \\
\hline 30 & 1.905 & 3.133 & 4.816 & 7.100 & 10.155 & 14.182 & 19.415 \\
\hline
\end{tabular}

Table 5 


\section{Games with More than Two Agents}

This section presents computational results pertaining to games with more than two agents. For such games the integral in the formula presented in Theorem 1 is estimated using Monte Carlo methods. Since the standard deviation of the estimate is proportional to the inverse of the square root of the sample size, it is impractical to strive for the sort of precision that results from the numerical integration methods used to generate the estimates presented in the last section. In this section all results are accompanied with standard errors, and while it is still possible to get a clear sense of the general properties of the means, it is not possible to attain the same degree of confidence, nor is it possible to discern whether the means grow with the steadiness apparent in Table 5.

\subsection{Properties of the Means}

In Section 3 we identified the following properties of the mean number of Nash equilibria (summing over all supports) which we rephrase as hypotheses pertaining to games with arbitrary numbers of players:

(H1) The expected number of Nash equilibria is increasing when the number of strategies for any agent increases, holding the numbers of strategies for the other agents fixed.

(H2) If the number of strategies for one player increases while the numbers of strategies for the other players are held fixed, the expected number of equilibria increases at a decreasing rate.

(H3) As one increases the numbers of strategies for two agents by the same amount, holding the numbers of strategies for the other agents fixed, the expected number of equilibria increases at an increasing rate.

(H4) The increase in the mean number of equilibria resulting from an increase of one agent's number of strategies by one is an increasing function of the numbers of strategies of the other agents.

(H5) If the numbers of strategies for two agents differ by at least two, then increasing by one the number of strategies of the agent with fewer strategies, while decreasing by one the number of strategies of the agent with more (holding the numbers of strategies of all other agents fixed) increases the expected number of equilibria.

Tables 6, 7, and 8 present the estimates for, respectively, three person games in which one agent has two and three pure strategies, and four person games in which two of the agents each have two pure strategies. Note that exact versions of these tables would be symmetric across the diagonal.

Support for hypothesis (H1) is very strong, both within each table, and comparing Table 6 to the corresponding entries in Tables 7 and 8 . On the other hand, the computational evidence is pretty strongly contrary to (H2), especially in the right hand columns (and bottom rows) of Tables 7 and 8. Hypothesis (H3) asserts that as we proceed along a diagonal path in the tables from upper left to lower right, the rate of increase should accelerate, and indeed this seems to be the case, in the sense that the estimates in the tables conform to it in every instance. Hypothesis (H4) is also strongly supported, with no violations in any one of the tables, and increasing differences are also observed in compar- 
ing Table 6 with Table 7. Insofar as a three person game may viewed as a four person game in which one of the agents is a "dummy," in the sense of having a single pure strategy, (H4) also predicts that Table 8 should have larger first differences than both Table 6 and Table 7 , as is the case. Hypothesis (H5) is in accord with every diagonal from lower left to upper right in the tables, and also in the sense that each entry of Table 8 is larger than the corresponding entry of Table 7 .

Summarizing, Hypotheses (H1) and (H3)-(H5) are supported by the numerical calculations, while Hypotheses (H2) is refuted.

Table 9 displays the mean numbers of Nash equilibria for games in which all agents have the same number of pure strategies. In addition to the subjective sense of very rapid growth of complexity, two points are of interest. First, for entries toward the lower right the means generally exceed the number of pure strategy vectors times the number of agents. Since the latter quantity is the number of payoffs in the game, it appears that the average number of Nash equilibria grows more rapidly than this measure of the "size" of the game. Second, the table clearly conveys the impression that the mean number of equilibria is greater when a large number of agents each have a small number of pure strategies than when the two numbers are reversed. As mentioned above, we may view a game with $n$ players as a game with $n^{\prime}>n$ players where $n^{\prime}-n$ of the players are dummies with a single pure stragegy. Viewed in this light, this observation is additional evidence for hypotheses (H5). 


\begin{tabular}{|c|c|c|c|c|c|c|}
\hline \multicolumn{7}{|c|}{$\begin{array}{l}\text { Mean Number of Nash Equilibria } \\
\text { for } 2 \times \mathbf{m} \times \mathbf{n} \text { Games } \\
\text { mean / (standard error) }\end{array}$} \\
\hline $\begin{array}{l}\text { Numbers of } \\
\text { Strategies }\end{array}$ & 2 & 3 & 4 & 5 & 6 & 7 \\
\hline 2 & $\begin{array}{l}1.98 \\
(0.02)\end{array}$ & $\begin{array}{l}2.41 \\
(0.03)\end{array}$ & $\begin{array}{l}2.69 \\
(0.03)\end{array}$ & $\begin{array}{l}3.04 \\
(0.04)\end{array}$ & $\begin{array}{l}3.19 \\
(0.04)\end{array}$ & $\begin{array}{l}3.38 \\
(0.05)\end{array}$ \\
\hline 3 & $\begin{array}{l}2.36 \\
(0.03)\end{array}$ & $\begin{array}{l}3.21 \\
(0.04)\end{array}$ & $\begin{array}{l}3.87 \\
(0.05)\end{array}$ & $\begin{array}{l}4.40 \\
(0.06)\end{array}$ & $\begin{array}{l}5.01 \\
(0.07)\end{array}$ & $\begin{array}{l}5.34 \\
(0.07)\end{array}$ \\
\hline 4 & $\begin{array}{l}2.73 \\
(0.03)\end{array}$ & $\begin{array}{l}3.88 \\
(0.05)\end{array}$ & $\begin{array}{l}4.87 \\
(0.06)\end{array}$ & $\begin{array}{l}5.91 \\
(0.08)\end{array}$ & $\begin{array}{l}6.50 \\
(0.08)\end{array}$ & $\begin{array}{l}7.47 \\
(0.10)\end{array}$ \\
\hline 5 & $\begin{array}{l}3.00 \\
(0.04)\end{array}$ & $\begin{array}{l}4.39 \\
(0.05)\end{array}$ & $\begin{array}{l}5.81 \\
(0.07)\end{array}$ & $\begin{array}{l}7.19 \\
(0.10)\end{array}$ & $\begin{array}{l}8.49 \\
(0.11)\end{array}$ & $\begin{array}{l}9.59 \\
(0.13)\end{array}$ \\
\hline 6 & $\begin{array}{l}3.23 \\
(0.05)\end{array}$ & $\begin{array}{l}4.93 \\
(0.06)\end{array}$ & $\begin{array}{l}6.62 \\
(0.09)\end{array}$ & $\begin{array}{l}8.42 \\
(0.11)\end{array}$ & $\begin{array}{l}10.12 \\
(0.13)\end{array}$ & $\begin{array}{l}11.91 \\
(0.17)\end{array}$ \\
\hline 7 & $\begin{array}{l}3.41 \\
(0.05)\end{array}$ & $\begin{array}{l}5.23 \\
(0.07)\end{array}$ & $\begin{array}{l}7.41 \\
(0.10)\end{array}$ & $\begin{array}{l}9.39 \\
(0.13)\end{array}$ & $\begin{array}{l}11.81 \\
(0.16)\end{array}$ & $\begin{array}{l}14.07 \\
(0.19)\end{array}$ \\
\hline 8 & $\begin{array}{l}3.56 \\
(0.05)\end{array}$ & $\begin{array}{l}5.75 \\
(0.08)\end{array}$ & $\begin{array}{l}8.08 \\
(0.11)\end{array}$ & $\begin{array}{l}11.13 \\
(0.15)\end{array}$ & $\begin{array}{l}13.41 \\
(0.19)\end{array}$ & $\begin{array}{l}16.13 \\
(0.22)\end{array}$ \\
\hline 9 & $\begin{array}{l}3.65 \\
(0.05)\end{array}$ & $\begin{array}{l}6.09 \\
(0.09)\end{array}$ & $\begin{array}{l}8.62 \\
(0.11)\end{array}$ & $\begin{array}{l}11.76 \\
(0.17)\end{array}$ & $\begin{array}{l}14.79 \\
(0.20)\end{array}$ & $\begin{array}{l}18.97 \\
(0.28)\end{array}$ \\
\hline 10 & $\begin{array}{l}3.74 \\
(0.06)\end{array}$ & $\begin{array}{l}6.30 \\
(0.09)\end{array}$ & $\begin{array}{l}9.26 \\
(0.12)\end{array}$ & $\begin{array}{l}12.67 \\
(0.18)\end{array}$ & $\begin{array}{l}16.74 \\
(0.25)\end{array}$ & $\begin{array}{l}20.75 \\
(0.28)\end{array}$ \\
\hline 11 & $\begin{array}{l}3.99 \\
(0.06)\end{array}$ & $\begin{array}{l}6.50 \\
(0.09)\end{array}$ & $\begin{array}{l}9.94 \\
(0.14)\end{array}$ & $\begin{array}{l}13.79 \\
(0.19)\end{array}$ & $\begin{array}{l}18.03 \\
(0.25)\end{array}$ & $\begin{array}{l}23.57 \\
(0.33)\end{array}$ \\
\hline 12 & $\begin{array}{l}3.96 \\
(0.06)\end{array}$ & $\begin{array}{l}6.77 \\
(0.09)\end{array}$ & $\begin{array}{l}10.49 \\
(0.17)\end{array}$ & $\begin{array}{l}15.22 \\
(0.23)\end{array}$ & $\begin{array}{l}19.42 \\
(0.29)\end{array}$ & $\begin{array}{l}25.70 \\
(0.36)\end{array}$ \\
\hline
\end{tabular}

Table 6 


\begin{tabular}{|c|c|c|c|c|c|c|}
\hline \multicolumn{7}{|c|}{$\begin{array}{l}\text { Mean Number of Nash Equilibria } \\
\text { for } \mathbf{3} \times \mathbf{m} \times \mathbf{n} \text { Games } \\
\text { mean / (standard error) }\end{array}$} \\
\hline $\begin{array}{l}\text { Numbers of } \\
\text { Strategies }\end{array}$ & 2 & 3 & 4 & 5 & 6 & 7 \\
\hline 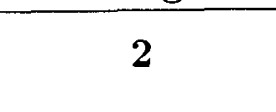 & $\begin{array}{l}2.44 \\
(0.03)\end{array}$ & $\begin{array}{l}3.17 \\
(0.04)\end{array}$ & $\begin{array}{l}3.90 \\
(0.05)\end{array}$ & $\begin{array}{l}4.50 \\
(0.06)\end{array}$ & $\begin{array}{l}4.91 \\
(0.06)\end{array}$ & $\begin{array}{l}5.30 \\
(0.07)\end{array}$ \\
\hline 3 & $\begin{array}{l}3.19 \\
(0.04)\end{array}$ & $\begin{array}{l}4.64 \\
(0.05)\end{array}$ & $\begin{array}{l}5.98 \\
(0.07)\end{array}$ & $\begin{array}{l}7.12 \\
(0.08)\end{array}$ & $\begin{array}{l}8.27 \\
(0.09)\end{array}$ & $\begin{array}{l}9.18 \\
(0.11)\end{array}$ \\
\hline 4 & $\begin{array}{l}3.79 \\
(0.05)\end{array}$ & $\begin{array}{l}5.90 \\
(0.07)\end{array}$ & $\begin{array}{l}7.94 \\
(0.09)\end{array}$ & $\begin{array}{l}10.11 \\
(0.12)\end{array}$ & $\begin{array}{l}11.94 \\
(0.14)\end{array}$ & $\begin{array}{l}13.45 \\
(0.16)\end{array}$ \\
\hline 5 & $\begin{array}{l}4.44 \\
(0.06)\end{array}$ & $\begin{array}{l}7.16 \\
(0.08)\end{array}$ & $\begin{array}{l}10.02 \\
(0.12)\end{array}$ & $\begin{array}{l}13.04 \\
(0.15)\end{array}$ & $\begin{array}{l}15.70 \\
(0.17)\end{array}$ & $\begin{array}{l}18.37 \\
(0.20)\end{array}$ \\
\hline 6 & $\begin{array}{l}4.92 \\
(0.06)\end{array}$ & $\begin{array}{l}8.17 \\
(0.10)\end{array}$ & $\begin{array}{l}11.80 \\
(0.14)\end{array}$ & $\begin{array}{l}15.69 \\
(0.17)\end{array}$ & $\begin{array}{l}19.89 \\
(0.22)\end{array}$ & $\begin{array}{l}23.84 \\
(0.25)\end{array}$ \\
\hline 7 & $\begin{array}{l}5.30 \\
(0.07)\end{array}$ & $\begin{array}{l}9.07 \\
(0.11)\end{array}$ & $\begin{array}{l}14.02 \\
(0.16)\end{array}$ & $\begin{array}{l}18.32 \\
(0.20)\end{array}$ & $\begin{array}{l}24.16 \\
(0.26)\end{array}$ & $\begin{array}{l}29.36 \\
(0.32)\end{array}$ \\
\hline 8 & $\begin{array}{l}5.73 \\
(0.08)\end{array}$ & $\begin{array}{l}10.08 \\
(0.12)\end{array}$ & $\begin{array}{l}15.35 \\
(0.18)\end{array}$ & $\begin{array}{l}21.94 \\
(0.24)\end{array}$ & $\begin{array}{l}27.87 \\
(0.30)\end{array}$ & $\begin{array}{l}35.28 \\
(0.38)\end{array}$ \\
\hline 9 & $\begin{array}{l}6.02 \\
(0.08)\end{array}$ & $\begin{array}{l}10.91 \\
(0.14)^{\circ}\end{array}$ & $\begin{array}{l}16.88 \\
(0.20)\end{array}$ & $\begin{array}{l}24.00 \\
(0.28)\end{array}$ & $\begin{array}{l}32.35 \\
(0.36)\end{array}$ & $\begin{array}{l}40.42 \\
(0.43)\end{array}$ \\
\hline 10 & $\begin{array}{l}6.38 \\
(0.09)\end{array}$ & $\begin{array}{l}12.06 \\
(0.15)\end{array}$ & $\begin{array}{l}18.62 \\
(0.22)\end{array}$ & $\begin{array}{l}27.52 \\
(0.33)\end{array}$ & $\begin{array}{l}36.16 \\
(0.41)\end{array}$ & $\begin{array}{l}47.98 \\
(0.53)\end{array}$ \\
\hline 11 & $\begin{array}{l}6.65 \\
(0.09)\end{array}$ & $\begin{array}{l}12.45 \\
(0.15)\end{array}$ & $\begin{array}{l}19.93 \\
(0.22)\end{array}$ & $\begin{array}{l}29.65 \\
(0.35)\end{array}$ & $\begin{array}{l}41.30 \\
(0.46)\end{array}$ & $\begin{array}{l}53.82 \\
(0.62)\end{array}$ \\
\hline 12 & $\begin{array}{l}6.87 \\
(0.10)\end{array}$ & $\begin{array}{l}13.22 \\
(0.17)\end{array}$ & $\begin{array}{l}21.35 \\
(0.26)\end{array}$ & $\begin{array}{l}31.84 \\
(0.36)\end{array}$ & $\begin{array}{l}45.20 \\
(0.51)\end{array}$ & $\begin{array}{l}59.04 \\
(0.61)\end{array}$ \\
\hline
\end{tabular}

Table 7 


\begin{tabular}{|c|c|c|c|c|c|c|}
\hline \multicolumn{7}{|c|}{$\begin{array}{l}\text { Mean Number of Nash Equilibria } \\
\text { for } 2 \times 2 \times \mathbf{m} \times \mathbf{n} \text { Games } \\
\text { mean / (standard error) }\end{array}$} \\
\hline $\begin{array}{l}\text { Numbers of } \\
\text { Strategies }\end{array}$ & 2 & 3 & 4 & 5 & 6 & 7 \\
\hline - & $\begin{array}{l}3.62 \\
(0.03)\end{array}$ & $\begin{array}{l}4.89 \\
(0.04)\end{array}$ & $\begin{array}{l}5.91 \\
(0.06)\end{array}$ & $\begin{array}{l}6.84 \\
(0.07)\end{array}$ & $\begin{array}{l}7.57 \\
(0.08)\end{array}$ & $\begin{array}{l}8.09 \\
(0.08)\end{array}$ \\
\hline 3 & $\begin{array}{l}4.83 \\
(0.04)\end{array}$ & $\begin{array}{l}7.07 \\
(0.06)\end{array}$ & $\begin{array}{l}9.11 \\
(0.08)\end{array}$ & $\begin{array}{l}10.90 \\
(0.10)\end{array}$ & $\begin{array}{l}12.57 \\
(0.12)\end{array}$ & $\begin{array}{l}13.89 \\
(0.13)\end{array}$ \\
\hline 4 & $\begin{array}{l}5.80 \\
(0.05)\end{array}$ & $\begin{array}{l}9.01 \\
(0.08)\end{array}$ & $\begin{array}{l}12.07 \\
(0.11)\end{array}$ & $\begin{array}{l}15.22 \\
(0.13)\end{array}$ & $\begin{array}{l}18.17 \\
(0.17)\end{array}$ & $\begin{array}{l}20.39 \\
(0.18)\end{array}$ \\
\hline 5 & $\begin{array}{l}6.75 \\
(0.07)\end{array}$ & $\begin{array}{l}11.02 \\
(0.10)\end{array}$ & $\begin{array}{l}15.04 \\
(0.13)\end{array}$ & $\begin{array}{l}19.38 \\
(0.17)\end{array}$ & $\begin{array}{l}24.07 \\
(0.22)\end{array}$ & $\begin{array}{l}28.21 \\
(0.25)\end{array}$ \\
\hline 6 & $\begin{array}{l}7.61 \\
(0.08)\end{array}$ & $\begin{array}{l}12.41 \\
(0.12)\end{array}$ & $\begin{array}{l}17.79 \\
(0.16)\end{array}$ & $\begin{array}{l}23.77 \\
(0.21)\end{array}$ & $\begin{array}{l}29.54 \\
(0.26)\end{array}$ & $\begin{array}{l}35.64 \\
(0.32)\end{array}$ \\
\hline 7 & $\begin{array}{l}8.30 \\
(0.09)\end{array}$ & $\begin{array}{l}14.01 \\
(0.13)\end{array}$ & $\begin{array}{l}20.76 \\
(0.19)\end{array}$ & $\begin{array}{l}27.76 \\
(0.26)\end{array}$ & $\begin{array}{l}35.96 \\
(0.33)\end{array}$ & $\begin{array}{l}44.20 \\
(0.41)\end{array}$ \\
\hline 8 & $\begin{array}{l}8.66 \\
(0.09)\end{array}$ & $\begin{array}{l}15.40 \\
(0.15)\end{array}$ & $\begin{array}{l}23.29 \\
(0.21)\end{array}$ & $\begin{array}{l}32.36 \\
(0.30)\end{array}$ & $\begin{array}{l}41.48 \\
(0.39)\end{array}$ & $\begin{array}{l}53.99 \\
(0.50)\end{array}$ \\
\hline 9 & $\begin{array}{l}9.37 \\
(0.11)\end{array}$ & $\begin{array}{l}16.96 \\
(0.17)^{\circ}\end{array}$ & $\begin{array}{l}25.61 \\
(0.24)\end{array}$ & $\begin{array}{l}35.92 \\
(0.32)\end{array}$ & $\begin{array}{l}48.36 \\
(0.45)\end{array}$ & $\begin{array}{l}62.54 \\
(0.58)\end{array}$ \\
\hline 10 & $\begin{array}{l}9.76 \\
(0.11)\end{array}$ & $\begin{array}{l}17.67 \\
(0.18)\end{array}$ & $\begin{array}{l}28.36 \\
(0.27)\end{array}$ & $\begin{array}{l}40.45 \\
(0.37)\end{array}$ & $\begin{array}{l}54.46 \\
(0.51)\end{array}$ & $\begin{array}{l}71.24 \\
(0.66)\end{array}$ \\
\hline 11 & $\begin{array}{l}10.29 \\
(0.12)\end{array}$ & $\begin{array}{l}19.53 \\
(0.21)\end{array}$ & $\begin{array}{l}30.61 \\
(0.30)\end{array}$ & $\begin{array}{l}44.78 \\
(0.44)\end{array}$ & $\begin{array}{l}60.85 \\
(0.54)\end{array}$ & $\begin{array}{l}79.74 \\
(0.77)\end{array}$ \\
\hline 12 & $\begin{array}{l}10.67 \\
(0.13)\end{array}$ & $\begin{array}{l}20.09 \\
(0.21)\end{array}$ & $\begin{array}{l}32.73 \\
(0.32)\end{array}$ & $\begin{array}{l}47.88 \\
(0.46)\end{array}$ & $\begin{array}{l}67.64 \\
(0.66)\end{array}$ & $\begin{array}{l}90.01 \\
(0.88)\end{array}$ \\
\hline
\end{tabular}

Table 8 


\begin{tabular}{|c|c|c|c|c|c|}
\hline \multicolumn{6}{|c|}{$\begin{array}{c}\text { Mean Number of Nash Equilibria } \\
\text { When } n \text { Agents Each Have } k \text { Strategies } \\
\text { mean / (standard error) }\end{array}$} \\
\hline & \multicolumn{5}{|c|}{ Common Number of Strategies } \\
\hline $\begin{array}{c}\text { Number of } \\
\text { Agents }\end{array}$ & 2 & 3 & 4 & 5 & 6 \\
\hline 2 & $\begin{array}{l}1.31 \\
(0.13)\end{array}$ & $\begin{array}{l}1.52 \\
(0.12)\end{array}$ & $\begin{array}{l}1.77 \\
(0.18)\end{array}$ & $\begin{array}{l}2.64 \\
(0.44)\end{array}$ & $\begin{array}{l}2.61 \\
(0.52)\end{array}$ \\
\hline 3 & $\begin{array}{l}2.15 \\
(0.20)\end{array}$ & $\begin{array}{l}3.76 \\
(0.33)\end{array}$ & $\begin{array}{l}12.66 \\
(1.13)\end{array}$ & $\begin{array}{l}27.23 \\
(2.78)\end{array}$ & $\begin{array}{l}65.69 \\
(4.19)\end{array}$ \\
\hline 4 & $\begin{array}{l}4.49 \\
(0.40)\end{array}$ & $\begin{array}{l}18.01 \\
(1.22)\end{array}$ & $\begin{array}{l}82.49 \\
(3.87)\end{array}$ & $\begin{array}{l}440.02 \\
(18.86)\end{array}$ & $\begin{array}{l}2036.54 \\
(65.98)\end{array}$ \\
\hline 5 & $\begin{array}{l}6.98 \\
(0.43)\end{array}$ & $\begin{array}{l}81.82 \\
(3.32)\end{array}$ & $\begin{array}{l}879.24 \\
(32.94)\end{array}$ & $\begin{array}{l}8826.17 \\
(177.63)\end{array}$ & $\begin{array}{l}90260.91 \\
(1580.96)\end{array}$ \\
\hline 6 & $\begin{array}{l}15.75 \\
(0.96)\end{array}$ & $\begin{array}{l}401.61 \\
(10.77)\end{array}$ & $\begin{array}{l}10086.00 \\
(207.80)\end{array}$ & $\begin{array}{l}243859.49 \\
(3320.24)\end{array}$ & $\begin{array}{l}5630939.33 \\
(50803.03)\end{array}$ \\
\hline
\end{tabular}

Table 9. 


\section{The Expected Number of Totally Mixed Equilibria}

We turn to the study of the expected number of totally mixed equilibria, so that throughout this section we assume that $T_{i}=S_{i}$ for all $i$. By virtue of Lemma A.3. the factors $r(a, b)$ in (2) are unity. Analytically, our focus is the expectation of the absolute value of the determinant of $\tilde{Z}_{\mathbf{p}}$. We will describe related work that leads to a relationship between the mean number of totally mixed equilibria and the square root of the maximal number. The resulting lower bounds on the mean are to some extent more tractable than the formula of Theorem 1, and in particular we are able to give a sense in which it can be proven that the expected number of totally mixed equilibria grows exponentially as the game becomes large.

Our discussion begins at a high level of generality, with a result from the theory of sparse systems of polynomial equations. We will work with polynomials in the variables $\mathbf{x}=\left(x_{1}, \ldots, x_{r}\right)$. For an exponent vector $a \in \mathbf{N}^{r}$ let $\mathbf{x}^{a}$ denote the monomial $x_{1}^{a_{1}}, \ldots, x_{r}^{a_{r}}$. A sparse system of polynomials is given by specifying supports $\mathcal{A}_{1}, \ldots, \mathcal{A}_{p} \subset$ $\mathbf{N}^{r}$, where each $\mathcal{A}_{k}$ is nonempty and finite, the interpretation being that we are studying the polynomial equation systems $f(\mathbf{x})=\left(f_{1}(\mathbf{x}), \ldots, f_{p}(\mathbf{x})\right)=0$ where, for $k=1, \ldots, p$, $f_{k}(\mathbf{x})=\sum_{a \in \mathcal{A}_{k}} f_{k a} \mathbf{x}^{a}$ is a polynomial with support contained in $\mathcal{A}_{k}$. For $k=1, \ldots, p$ let $\mathcal{H}_{k}:=\mathbb{R}^{\mathcal{A}_{k}}$ be the space of real coefficient vectors $f_{k}=\left(f_{k a}\right)_{a \in \mathcal{A}_{k}}$, and let

$$
\mathcal{H}=\mathcal{H}_{1} \times \ldots \times \mathcal{H}_{p} .
$$

Sparse systems have been studied very intensively in recent years, in part because computational advances have led to a situation in which algorithmic exploitation of sparsity has practical applications, but also in large part because of the following theorem of Bernshtein (1975) and the methods used to prove it. The Newton polytope of $f_{k}$ is the convex polytope $Q_{k}=\operatorname{con}\left(\mathcal{A}_{k}\right) \subset \mathbb{R}^{r}$. Assume that there are as many equations as unknowns: $r=p$. The mixed volume of $Q_{1}, \ldots, Q_{p}$, which was first defined and studied by Minkowski, and which we denote by $\mathcal{M V}\left(Q_{1}, \ldots, Q_{p}\right)$, may be defined to be the coefficient of the monomial $\lambda_{1} \cdot \ldots \cdot \lambda_{p}$ in the polynomial $\operatorname{vol}\left(Q_{\lambda}\right)$, where

$$
Q_{\lambda}=\lambda_{1} Q_{1}+\ldots+\lambda_{p} Q_{p} .
$$

(See Ewald (1996) for a proof that $\operatorname{vol}\left(Q_{\lambda}\right)$ is, in fact, a polynomial.)

Theorem: (Bernshtein (1975)) Let $\mathcal{H}^{\mathbf{C}}=\mathcal{H}_{1}^{\mathbf{C}} \times \ldots \times \mathcal{H}_{p}^{\mathbf{C}}$ where $\mathcal{H}_{k}^{\mathbf{C}}=\mathbf{C}^{\mathcal{A}_{k}}$ is the space of complex polynomials with support $\mathcal{A}_{k}$. For systems $f$ in the complement, in $\mathcal{H}^{\mathrm{C}}$, of an algebraic set of positive (complex) codimension, there are $\mathcal{M V}\left(Q_{1}, \ldots, Q_{p}\right)$ roots in $\left(\mathbf{C}^{*}\right)^{p}$, where $\mathbf{C}^{*}:=\mathbf{C} \backslash\{0\}$.

In recognition of closely related work (Kushnirenko (1975), Khovanski (1978)) the generic number of roots is sometimes called the $B K K$ bound.

We say that the sparse system is multihomogeneous if the following description is satisfied: the variables in $\mathbf{x}$ are grouped in $n$ blocks $\left(\mathbf{y}_{1}, \ldots, \mathbf{y}_{n}\right)$, where $\mathbf{y}_{i}=\left(y_{i 0}, y_{i 1}, \ldots, y_{i p_{i}}\right)$, and, for a given $p \times n$ matrix $\delta$ of nonnegative integers, each $\mathcal{A}_{k}$ represents the set of polynomials that are homogeneous of degree $\delta_{k i}$ as a function of $\mathbf{y}_{i}, i=1, \ldots, n$. In combinatoric terms this means that

$$
\begin{gathered}
\mathcal{A}_{k}=\mathcal{A}_{k 1} \times \ldots \times \mathcal{A}_{k n} \\
-20-
\end{gathered}
$$


where $\mathcal{A}_{k i}$ is the simplex (in the integer lattice) of coefficient vectors in $\mathbf{N}^{p_{i}+1}$ that sum to $\delta_{k i}$.

If $\left(\mathbf{y}_{1}, \ldots, \mathbf{y}_{n}\right)$ is a root of the multihomogeneous system $f=0$, then, for any scalars $\alpha_{1}, \ldots, \alpha_{n}$, the point $\left(\alpha_{1} \mathbf{y}_{1}, \ldots, \alpha_{n} \mathbf{y}_{n}\right)$ is also a solution, so it is natural to look for solutions in $N:=S^{p_{1}} \times \ldots \times S^{p_{n}}$, where each $N_{i}:=S^{p_{i}}$ is the unit sphere in $\mathbb{R}^{p_{k}+1}$. An even more natural solution space is the cartesian product

$$
P:=P_{1} \times \ldots \times P_{n}
$$

of the projective spaces $P_{i}=\mathbf{P}\left(\mathbb{R}^{p_{i}+1}\right)$, which are obtained from the $N_{i}$ by identifying antipodal points. If the equation $f(\mathbf{x})=0$ holds at any of the $2^{n}$ points in $N$ identified with $z \in P$, then, by virtue of multihomogeneity, it holds at all the others as well. and we will follow an abuse of notation that is standard in algebraic geometry, writing ' $f(z)=0$ ' to express this condition, and describing $z$ as a root of $f$, even though $f$ is not a function defined on $P$.

We say that this system is exactly determined if there are, effectively, as many equations as degrees of freedom:

$$
p=p_{1}+\ldots+p_{n}=r-n
$$

Henceforth we consider only this case. Note that, with this restriction, the system is determined by $\mathbf{p}:=\left(p_{1}, \ldots, p_{n}\right)$ and $\delta$. In applying Bernshtein's theorem to multihomogeneous systems the natural procedure is to pass to the "demultihomogenized" system obtained by specializing the variables $y_{10}, \ldots, y_{n 0}$ to unity. This is the sparse system whose supports are the projections of $\mathcal{A}_{1}, \ldots, \mathcal{A}_{p}$ to the space of the variables

$$
y_{11}, \ldots, y_{1 p_{1}}, \ldots, y_{n 1}, \ldots, y_{n p_{n}}
$$

Let $\operatorname{BKK}(\mathbf{p}, \delta)$ be the generic number of complex roots of the demultihomogenized system, as given by Bernshtein's theorem.

The permanent (e.g., Egorychev (1996)) of a $p \times p$ matrix $D$ with entries $d_{k m}$ is defined to be

$$
\operatorname{per}(D):=\sum_{\sigma \in S_{p}}\left(\prod_{k=1}^{p} d_{k \sigma(k)}\right)
$$

(Hêre $S_{p}$ is the symmetric group, that is, the group of permutations of $\{1, \ldots, p\}$.) This formula differs from the one defining the determinant in that here each summand is not multiplied by $(-1)^{\operatorname{sgn}(\sigma)}$, and the permanent has several properties that are analogous to those of the determinant, such as multilinearity and the possibility of expanding by minors along any row or column.

Let $\Delta(\mathbf{p}, \delta)$ be the $p \times p$ matrix whose first $p_{1}$ columns are the first column of $\delta$, whose next $p_{2}$ columns are the second column of $\delta$, and so forth. The following results are proved in McLennan (1998a); the formula expressing the generic number of roots in terms of the permanent can be derived by expanding $\operatorname{vol}\left(Q_{\lambda}\right)$, and the two recursive formulas are derived by expanding the permanent by minors along a row or column respectively. 
We adopt the convention that $\operatorname{BKK}((0, \ldots, 0), \delta)=1$. That is, the "null system" with no variables and no equations has one root. For $i=1, \ldots, n$ let $\mathbf{e}_{i}$ denote the $i^{\text {th }}$ unit basis vector of $\mathbb{R}^{n}$.

\section{Proposition 5.1:}

$$
\operatorname{BKK}(\mathbf{p}, \delta)=\frac{\operatorname{per}(\Delta(\mathbf{p}, \delta))}{p_{1} ! \cdot \ldots \cdot p_{n} !} .
$$

For $k=1, \ldots, p$ let $\delta^{-k}$ be the $(p-1) \times n$ matrix obtained by eliminating the $k^{\text {th }}$ row of $\delta$. Then

$$
\operatorname{BKK}(\mathbf{p}, \delta)=\sum_{i: p_{i}>0} \delta_{k i} \cdot \operatorname{BKK}\left(\mathbf{p}-\mathbf{e}_{i}, \delta^{-k}\right)
$$

for all $k=1, \ldots, p$, and

$$
\operatorname{BKK}(\mathbf{p}, \delta)=\frac{1}{p_{i}} \sum_{k=1}^{p} \delta_{k i} \cdot \operatorname{BKK}\left(\mathbf{p}-\mathbf{e}_{i}, \delta^{-k}\right)
$$

for all $i=1, \ldots, n$ such that $p_{i}>0$.

McLennan (1998b) studies the mean number of roots of a random multihomogeneous system of equations, with respect to a model of a random system derived from the following inner product. Endow each space of real coefficient vectors $\mathcal{H}_{k}$ with the inner product

$$
\left\langle f_{k}, f_{k}^{\prime}\right\rangle_{k}:=\sum_{a \in \mathcal{A}_{k}} \zeta(a) f_{k a} f_{k a}^{\prime}
$$

where

$$
\zeta(a):=\frac{a_{10} ! \cdot \ldots \cdot a_{1 p_{1}} !}{\left(a_{10}+\ldots+a_{1 p_{1}}\right) !} \cdot \ldots \cdot \frac{a_{n 0} ! \cdot \ldots \cdot a_{n p_{n}} !}{\left(a_{n 0}+\ldots+a_{n p_{n}}\right) !} .
$$

In McLennan (1998b) this is shown to be the unique inner product such that:

(a) the 1-dimensional coordinate subspaces corresponding to the various monomials in $\mathcal{H}_{k}$ are orthogonal; and

(b) the inner product is invariant under the action of $O\left(\mathbb{R}^{p_{1}+1}\right) \times \ldots \times O\left(\mathbb{R}^{p_{n}+1}\right)$ given by $h f_{k}=f_{k} \circ\left(h_{1}, \ldots, h_{n}\right)$. (Recall that $O(m)$ is the group of orthogonal (inner product preserving) linear automorphisms of $\mathbb{R}^{m}$.)

Let $\mathcal{S}_{k}$ be the unit sphere, with respect to this inner product, in $\mathcal{H}_{k}$. The uniform distribution on

$$
\mathcal{S}:=\mathcal{S}_{1} \times \ldots \times \mathcal{S}_{n}
$$

is our model of a random multihomogeneous system. Let $\lambda$ be the measure on $P$ defined, for measurable $E \subset P$, by

$$
\lambda(E)=\int_{\mathcal{S}} \#(\{z \in E: f(z)=0\}) d \mathbf{U}_{\mathcal{S}}(f) .
$$


Denote the expected number of roots in $P$ by

$$
E(\mathbf{p}, \delta):=\lambda(P)
$$

Let $\tilde{Z}_{(\mathbf{p}, \delta)}$ be a random $p \times p$ matrix whose entries $\tilde{z}_{k}^{i j}$ are independently distributed normal random variables with mean zero and variance $\delta_{k i}$. The next section proves Proposition 2.1 by displaying it as a consequence of the following result.

Proposition 5.2: (McLennan (1998b)) For all measurable $E \subset P$

$$
\lambda(E)=\mathbf{U}_{P}(E) \cdot 2^{-p / 2} \cdot\left(\prod_{i=1}^{n} \frac{\Gamma\left(\frac{1}{2}\right)}{\Gamma\left(\frac{p_{i}+1}{2}\right)}\right) \cdot \mathbf{E}\left(\left|\operatorname{det} \tilde{Z}_{(\mathbf{p}, \delta)}\right|\right) .
$$

In particular,

$$
E(\mathbf{p}, \delta)=2^{-p / 2} \cdot\left(\prod_{i=1}^{n} \frac{\Gamma\left(\frac{1}{2}\right)}{\Gamma\left(\frac{p_{i}+1}{2}\right)}\right) \cdot \mathbf{E}\left(\left|\operatorname{det} \tilde{Z}_{(\mathbf{p}, \delta)}\right|\right)
$$

The next two results give bounds on $E(\mathbf{p}, \delta)$. The first is more powerful, in that it implies the second by induction.

Proposition 5.3: (McLennan (1998b)) For all $k=1, \ldots, p$,

$$
\sum_{i: p_{i}>0} \sqrt{\delta_{k i}} \cdot E\left(\mathbf{p}-\mathbf{e}_{i}, \delta^{-k}\right) \geq E(\mathbf{p}, \delta) \geq \sqrt{\sum_{i: p_{i}>0} \delta_{k i} \cdot E\left(\mathbf{p}-\mathbf{e}_{i}, \delta^{-k}\right)^{2}} .
$$

Both of these inequalities hold strictly if and only if there is more than one $i$ with $p_{i}>0$ and $\delta_{k i}>0$.

In preparation for the next result we ask when it is the case that $\operatorname{BKK}(\mathbf{p}, \delta)$ can be computed by repeated applications of this inequality in which the RHS has only one nonzero term, so that the relation holds with equality. We say that the pair $(\mathbf{p}, \delta)$ is simply reducible if the following inductive definition is satisfied: there is some $k$ for which there is at most one $i$ with $n_{i}>0, \delta_{k i}>0$, and $\operatorname{BKK}\left(\mathbf{p}-\mathbf{e}_{i}, \delta^{-k}\right)>0$, and if $p>1$ we require that for this $i,\left(\mathbf{p}-\mathbf{e}_{i}, \delta^{-k}\right)$ is also simply reducible.

- Let $\Delta_{\frac{1}{2}}(\mathbf{p}, \delta)$ be the $p \times p$ matrix whose $(k, i h)$-entry is $\sqrt{\delta_{k i}}$. Both of the inequalities above are comparable to the first recursive equation in Proposition 5.1. By au induction on $p$ that begins with the convention that $E(\mathbf{p}, \delta)=\operatorname{BKK}(\mathbf{p}, \delta)=1$ when $p_{1}=\ldots=p_{k}=0$, and whose induction step is a matter of combining the inequality above with the result for the various ( $\mathbf{p}-\mathbf{e}_{i}, \delta^{-k}$ ), which may be assumed by induction, they imply:

Proposition 5.4: (McLennan (1998b))

$$
\frac{\operatorname{per} \Delta_{\frac{1}{2}}(\mathbf{p}, \delta)}{p_{1} ! \cdot \ldots \cdot p_{k} !} \geq E(\mathbf{p}, \delta) \geq \sqrt{\frac{\operatorname{per} \Delta(\mathbf{p}, \delta)}{p_{1} ! \cdot \ldots \cdot p_{k} !}}
$$


Each of these inequalities holds with equality if and only if $(\mathbf{p}, \delta)$ is simply reducible. In particular (by virtue of Proposition 5.1)

$$
E(\mathbf{p}, \delta) \geq \sqrt{\operatorname{BKK}(\mathbf{p}, \delta)}
$$

with equality if and only if $(\mathbf{p}, \delta)$ is simply reducible.

We now turn to the systems arising from normal form games. Set

$$
\Delta(\mathbf{p}):=\Delta(\mathbf{p}, \delta(\mathbf{p})), \quad \operatorname{BKK}(\mathbf{p}):=\operatorname{BKK}(\mathbf{p}, \delta(\mathbf{p})), \quad E(\mathbf{p}):=E(\mathbf{p}, \delta(\mathbf{p})) .
$$

Note that $\Delta_{\frac{1}{2}}(\mathbf{p}, \delta)=\Delta(\mathbf{p}, \delta)$ when each $\delta_{i j}$ is either 0 or 1 , as is the case with $\Delta(\mathbf{p})$. In view of Proposition 5.1, the upper bound given by the last result reduces to $\operatorname{BKK}(\mathbf{p}) \geq E(\mathbf{p})-$ the generic number of complex roots is at least as large as the mean number of real rootswhich is already obvious, of course. For normal form games, therefore, the significance of the last result is entirely a matter of the lower bound on $E(\mathbf{p})$. In general, our methods provide upper bounds on the mean number of totally mixed equilibria only through the finding that the roots of the relevant system are uniformly distributed on $N$, so that the fraction of them lying in $N^{++}$is obtained by dividing by $2^{p+n}$.

The main advantage of the inequality above over the formula given by the Corollary to Theorem 1 is that it is combinatoric in nature. Going further in the direction of trading accuracy for transparency and ease of evaluation, there are the following bounds, which are rather crude, but useful nonetheless. They are derived by an inductive argument based on the recursive formulas in Proposition 5.1.

Proposition 5.5: (McKelvey and McLennan (1997)) $\mathrm{BKK}(\mathbf{p})>0$ if and only if $p_{i} \leq$ $\sum_{j \neq i} p_{j}$ for all $i$. If this is the case, and $p_{1} \leq p_{2} \leq \ldots \leq p_{n}$, then

$$
(n-1)^{p} \geq \operatorname{BKK}(\mathbf{p}) \geq \prod_{i=1}^{n-1}(n-i)^{p_{i}} .
$$

The following, which is derived by combining the last result, the Corollary to Theorem 1, Proposition 5.2, and Proposition 5.4, summarizes the results to this point.

Theorem 2: If $p_{1} \leq p_{2} \leq \ldots \leq p_{n}$ and $p_{1}+\ldots+p_{n-1} \geq p_{n}$, then

$$
(n-1)^{p} \geq \operatorname{BKK}(\mathbf{p}) \geq \frac{2^{p} \cdot \tilde{\nu}\left(N^{++}\right)}{\prod_{i=1}^{n} r\left(p_{i},\left|S_{i} \backslash T_{i}\right|\right)} \geq \sqrt{\operatorname{BKK}(\mathbf{p})} \geq \sqrt{\prod_{i=1}^{n-1}(n-i)^{p_{i}}}
$$

McKelvey and McLennan (1997) show that for any normal form it is possible to find utilities for which there are as many regular totally mixed Nash equilibria as are allowed by 
Bernshtein's theorem. (McLennan (1998a) extends this result to general multihomogeneous systems.) In particular, the mean number of totally mixed Nash equilibria is greater than or equal to $2^{-p}$ times the square root of the maximal number of totally mixed Nash equilibria.

Theorem 2 has a variety of implications for the asymptotic growth rates of the mean number of equilibria. To begin with, we remark that the growth of the vector $\mathbf{p}$ must be controlled rather carefully. In particular, if we allow one agent's set of pure strategies to grow while holding the numbers of pure strategies for the other agents fixed. then eventually $\operatorname{BKK}(\mathbf{p})=E(\mathbf{p})=0$. Consequently the interesting questions concern the rates of growth as the numbers of pure strategies for at least two agents become large.

Since $\sqrt{\prod_{i=1}^{n-1}(n-i)^{p_{i}}}$ depends only on $p_{1}, \ldots, p_{n-2}$, this quantity is constant if we allow the numbers of pure strategies for two agents to increase together, while the numbers of pure strategies for all other agents are held fixed. In general, if we fix the numbers of pure strategies for some set of agents, letting the numbers of pure strategies for the other agents increase, the quantity $\prod_{i=1}^{n-1}(n-i)^{p_{i}}$ is a constant times what it would be if the fixed agents were not present at all.

The lower bound on $E(\mathbf{p})$ has the greatest force when all agents have the same number of pure strategies: $p_{1}=\ldots=p_{n}=p / n$. In this case the mean number of equilibria is bounded below by

$$
\left(\frac{(n-1) !}{2^{2 n}}\right)^{p / 2 n}
$$

For integral $n$ the quantity $(n-1) ! / 2^{2 n}$ exceeds one if and only if $n \geq 12$, so that the number of equilibria grows exponentially in this case. On the other hand, when $n \leq 3$ the upper bound in Theorem 2 implies that mean number of totally mixed equilibria is less than or equal to one. For $n$ between 4 and 11 we are unable to determine whether the asymptotic growth of the mean number of totally mixed equilibria is exponential.

\section{The Proof of Proposition 2.1}

This and the next section constitute the proof of Theorem 1. In this section we explain how Proposition 2.1 follows from Proposition 5.2. We begin with two issues that are easily dealt with.

As Remark 3 pointed out, the measure $\nu$ is unaffected by the sets $S_{i} \backslash T_{i}$. This means that, in proving Proposition 2.1, there is no loss of generality in assuming that $T_{i}=S_{i}$, and we shall do so throughout this section.

- The formulas given by Proposition 2.1 and Proposition 5.2 differ by a factor of $2^{n}$ that arises from the fact that $\nu$ is the measure induced on $N$ while $\lambda$ is a measure on $P$. Let $\kappa$ be the measure on $N$ defined, for measurable $E \subset N$, by

$$
\kappa(E)=\int_{\mathcal{S}} \#(\{z \in E: f(z)=0\}) d \mathbf{U}_{\mathcal{S}}(f) .
$$

Letting $\rho: N \rightarrow P$ be the natural map from $N$ to $P$, for $E \subset N$ such that the restriction of $\rho$ to $E$ is injective we clearly have $\kappa(E)=\lambda(E)$, so $\kappa\left(\rho^{-1}(F)\right)=2^{n} \cdot \lambda(F)$ for any measurable $F \subset P$. Clearly Proposition 2.1 follows from Proposition 5.2 once we show that $\nu=\kappa$. 
The measures $\nu$ and $\kappa$ are the induced distributions of roots of two random systems of polynomials that have the same support. For each agent $i$ let $T_{-i}:=\prod_{h \neq i} T_{h}$. There are $p_{i}$ equations whose common support is the set of monomials of the form $\prod_{h \neq i} \tau_{h}\left(t_{h}\right)$ for $t_{-i} \in T_{-i}$. In the notation of Section 3 , for each $i=1, \ldots, n$ there are $p_{i}$ indices $k$ such that $\delta_{k i}=0$ and $\delta_{k h}=1$ for all $h \neq i$.

We now describe the distribution of coefficient vectors generated by the equations of totally mixed equilibrium. Let $\Theta_{i}: \mathbb{R}^{T_{1}} \times \ldots \times \mathbb{R}^{T_{n}} \rightarrow \mathbb{R}^{T_{-i}}$ be the function with $t_{-i}$-coordinate function

$$
\Theta_{i t_{-i}}(\tau)=\prod_{h \neq i} \tau_{h}\left(t_{h}\right)
$$

For $u_{i} \in\left(\mathbb{R}^{S}\right)^{*}$ and $s_{i} \in T_{i}$, let $u_{i}\left(s_{i}, \cdot\right) \in\left(\mathbb{R}^{T_{-i}}\right)^{*}$ be the linear functional obtained by restricting $u_{i}$ to $\mathbb{R}^{\left\{s_{i}\right\} \times T_{-i}}$. The equation expressing the indifference of agent $i$ between two pure strategies $t_{i}, t_{i}^{\prime} \in T_{i}$ may now be written as

$$
\left\langle u_{i}\left(t_{i}, \cdot\right)-u_{i}\left(t_{i}^{\prime}, \cdot\right), \Theta_{i}(\tau)\right\rangle=0 .
$$

Defining $\phi(u, \cdot) \in \mathcal{S}$ by setting

$$
\phi_{i j}(u, \tau)=\left\langle\frac{u_{i}\left(t_{i}^{j}, \cdot\right)-u_{i}\left(t_{i}^{0}, \cdot\right)}{\left\|u_{i}\left(t_{i}^{j}, \cdot\right)-u_{i}\left(t_{i}^{0}, \cdot\right)\right\|}, \Theta_{i}(\tau)\right\rangle
$$

we have map $u \mapsto \phi(u, \cdot)$ from $M$ to $\mathcal{S}$. If the measure induced from the uniform distribution on $M$ by this map was the uniform distribution, we would be done, but in fact this is almost certainly not the case ${ }^{(5)}$. In particular, the coefficients of $\phi_{i 1}(u, \cdot), \ldots, \phi_{i p_{i}}(u, \cdot)$ are all affected by $u_{i}\left(t_{i}^{0}, \cdot\right)$, so it would be quite surprising if they were distributed independently.

To handle this problem we pass to a more fundamental view of the two random systems of equations. Let $\Gamma_{i}$ be the Grassman manifold of planes of codimension $p_{i}$ in $\mathbb{R}^{T_{-i}}$, and let $\Gamma:=\Gamma_{1} \times \ldots \times \Gamma_{n}$. Let $M_{i}^{*}$ be the set of $u_{i} \in M_{i}$ such that

$$
u_{i}\left(t_{i}^{1}, \cdot\right)-u_{i}\left(t_{i}^{0}, \cdot\right), \ldots, u_{i}\left(t_{i}^{p_{i}}, \cdot\right)-u_{i}\left(t_{i}^{0}, \cdot\right)
$$

are linearly independent, and let

$$
M^{*}=M_{1}^{*} \times \ldots \times M_{n}^{*} .
$$

This linear independence holds generically, so $M^{*}$ is a set of full measure in $M$. Define $\gamma_{i}: M_{i}^{*} \rightarrow \Gamma_{i}$ by letting $\gamma_{i}\left(u_{i}\right)$ be the intersections of the kernels of

$$
u_{i}\left(t_{i}^{1}, \cdot\right)-u_{i}\left(t_{i}^{0}, \cdot\right), \ldots, u_{i}\left(t_{i}^{p_{i}}, \cdot\right)-u_{i}\left(t_{i}^{0}, \cdot\right),
$$

and for $u \in M$ set $\gamma(u)=\left(\gamma_{1}\left(u_{1}\right), \ldots, \gamma_{n}\left(u_{n}\right)\right)$ Then

$$
(u, \tau) \in V \Leftrightarrow(\forall i) \Theta_{i}(\tau) \in \gamma_{i}\left(u_{i}\right),
$$

(5) I do not actually have a proof of this. 
so the measure $\nu$ is entirely determined by the measure $\mathrm{U}_{M} \circ \gamma^{-1}$ on $\Gamma$. Since $u_{1}, \ldots, u_{n}$ are statistically independent, so are $\gamma_{1}\left(u_{1}\right), \ldots, \gamma_{n}\left(u_{n}\right)$, so in fact $\nu$ is determined by the measures on $\Gamma_{i}$ induced by $\gamma_{1}, \ldots, \gamma_{n}$.

The description of the measure on $\Gamma$ induced by a random coefficient vector in $\mathcal{S}$ is similar. For each $i$ let $\mathcal{S}^{i}=\prod_{k: \mathcal{A}_{k}=T_{-i}} \mathcal{S}_{k}$, and let $\mathcal{S}^{i *}$ be the set of $f^{i}=\left(f_{k}\right)_{\mathcal{A}_{k}=T_{-i}} \in \mathcal{S}^{i}$ such that the components $f_{k}$ of $f^{i}$ are linearly independent. Let $\mathcal{S}^{*}=\mathcal{S}^{1 *} \times \ldots \times \mathcal{S}^{n *}$ Define $\eta_{i}: \mathcal{S}^{i *} \rightarrow \Gamma_{i}$ by letting $\eta_{i}\left(f^{i}\right)$ be the intersection of the orthogonal complements of the components of $f^{i}$, and define $\eta: \mathcal{S} \rightarrow \Gamma$ by $\eta(f):=\left(\eta_{1}\left(f^{1}\right), \ldots, \eta_{n}\left(f^{n}\right)\right)$. Then $f(\tau)=0$ if and only if $\Theta_{i}(\tau) \in \eta_{i}(f)$ for all $i$. As above, the measure $\mu$ is entirely determined by the measure $\mathrm{U}_{\mathcal{S}} \circ \eta^{-1}$ on $\Gamma$. Since $f_{1}, \ldots, f_{p}$ are statistically independent, so are $\eta_{1}(f), \ldots, \eta_{n}(f)$, so in fact $\mu$ is determined by the measures on $\Gamma_{1}, \ldots, \Gamma_{n}$ induced by $\eta_{1}, \ldots, \eta_{n}$. Our goal now is to show that, for each $i$,

$$
\mathbf{U}_{M_{i}} \circ \gamma_{i}^{-1}=\mathbf{U}_{\mathcal{S}^{i}} \circ \eta_{i}^{-1}
$$

Our strategy will be to show that both these measures are invariant under a particular group action that admits only one invariant measure. Recall that a group $H$ acts (from the left) on a space $Y$ if there is a rule associating an element $h y \in Y$ to each pair $(h, y) \in Y$, such that:

(a) $e y=y$ for all $y \in Y$, where $e$ is the identity element of $H$;

(b) $h\left(h^{\prime} y\right)=\left(h h^{\prime}\right) y$ for all $h, h^{\prime} \in H$ and $y \in Y$.

The action is said to be transitive if for each $y, y^{\prime} \in Y$ there is some $h \in H$ with $h y=y^{\prime}$. If $H$ also acts on $Z$, a map $F: Y \rightarrow Z$ is equivariant if $F(h y)=h F(y)$ for all $y \in Y$ and $h \in H$.

Let $O\left(\mathbb{R}^{T_{-i}}\right)$ be the orthogonal group of $\mathbb{R}^{T_{-i}}$. This group acts from the left on $\Gamma_{i}$ in the obvious manner: for $K_{i} \in \Gamma_{i}$ and $h \in O\left(\mathbb{R}^{T_{-i}}\right)$ we set $h K_{i}=\left\{h z: z \in K_{i}\right\}$. It also acts on $M_{i}$ according to the formula

$$
h u_{i}=\left(u_{i}\left(t_{i}^{0}, \cdot\right) \circ h^{-1}, u_{i}\left(t_{i}^{1}, \cdot\right) \circ h^{-1}, \ldots, u_{i}\left(t_{i}^{p_{1}}, \cdot\right) \circ h^{-1}\right) .
$$

It is straightforward to check that, with respect to these actions, $\gamma_{i}$ is equivariant. Since $\mathbf{U}_{M_{i}}$ is invariant under this action, it follows that the induced distribution $\mathbf{U}_{M_{i}} \circ \gamma_{i}^{-1}$ on $\Gamma_{i}$ is also invariant.

Similarly, there is an action of $O\left(\mathbb{R}^{T_{-i}}\right)$ on $\mathcal{S}^{i *}$ in which a group element acts simultaneously on each component $f_{k} \in \mathbb{R}^{T_{-i}}$ of $f^{i}$. The uniform distribution on $\mathcal{S}^{i}$ is invariant under this action, and $\eta_{i}$ is equivariant, so that $\mathbf{U}_{\mathcal{S}^{i}} \circ \eta_{i}^{-1}$ is invariant.

Since $O\left(\mathbb{R}^{T_{-i}}\right)$ acts transitively on $\Gamma_{i}$, up to scalar multiplication there is exactly one invariant measure on $\Gamma_{i}$ (e.g., Nachbin $\left(1965\right.$, p. 138)) so $\mathbf{U}_{M_{i}} \circ \gamma_{i}^{-1}=\mathrm{U}_{\mathcal{S}^{i}} \circ \eta_{i}^{-1}$ as desired. This completes the proof of Proposition 2.1.

\section{The Proof of Proposition 2.2}

This section completes the proof of Theorem 1 by proving Proposition 2.2. The proof is fairly lengthy, paralleling many of the steps in the argument of McLennan (1998b), so it is divided into a number of shorter steps. 


\subsection{Manifold Theoretic Properties}

We begin by showing that the objects of study are well behaved from the point of view of the concepts of differential topology. To this end we describe $V$ in terms of a system of equations. For each $i$ and $j=1, \ldots, p_{i}$ define the real valued function $f_{i j}$ on $\left(\mathbb{R}^{S}\right)^{n} \times \prod_{i} \mathbb{R}^{T_{i}}$ by

$$
f_{i j}(u, \tau)=\left\langle u_{i}, \Theta\left(\tau \mid t_{i}^{j}\right)-\Theta\left(\tau \mid t_{i}^{0}\right)\right\rangle=\left\langle u_{i}\left(t_{i}^{j}, \cdot\right)-u_{i}\left(t_{i}^{0}, \cdot\right), \Theta_{i}\left(\tau_{-i}\right)\right\rangle .
$$

For each $i$ set $f_{i}=\left(f_{i 1}, \ldots, f_{i p_{i}}\right)$, and let $f=\left(f_{1}, \ldots, f_{n}\right)$. Then

$$
V=(M \times N) \cap f^{-1}(0) .
$$

Proposition 7.1: $V$ is a smooth manifold of the same dimension as $M$.

Proof: It is easy to verify that all points in $\left(\mathbb{R}^{S}\right)^{n} \times \prod_{i}\left(\mathbb{R}^{T_{i}} \backslash\{0\}\right)$ are regular points of $f$; the intuition is that at any $\tau$ with no $\tau_{i}$ vanishing, $\Theta_{i}\left(\tau_{-i}\right) \neq 0$, so the component function $f_{i j}$ can be varied freely by varying $u_{i}\left(t_{i}^{j}, \cdot\right)$. Fix a point $(u, \tau) \in V$. We wish to show that $(u, \tau)$ is also a regular point of the restriction of $f$ to $M \times N$. Let $(v, \xi)$ denote a vector in $\left(\mathbb{R}^{S}\right)^{n} \times \prod_{i} \mathbb{R}^{S_{i}}$, thought of as a tangent vector at $(u, \tau)$. If each $v_{i}$ is a scalar multiple of $u_{i}$ and each $\xi_{i}$ is scalar multiple of $\tau_{i}$, then, by virtue of the multihomogeneity of the formula defining $f$,

$$
f(u+\alpha v, \tau+\alpha \xi)=0
$$

for all $\alpha \in \mathbb{R}$, so the derivative of $f$ at $(u, \tau)$, applied to $(v, \xi)$, is zero. Consequently, if $D f(u, \tau)$ is surjective, so is the restriction of $D f(u, \tau)$ to $T_{(u, \tau)}(M \times N)$, which consists of those vectors $(v, \xi)$ with $v_{i}$ orthogonal to $u_{i}$ and $\xi_{i}$ orthogonal to $\tau_{i}$ for each $i$. Thus each point in $V$ is a regular point of the restriction of $f$ to $M \times N$, which means that 0 is a regular value of this restriction, and the claim now follows from the regular value theorem. (E.g., Guillemin and Pollack (1965).)

The fiber over $\tau \in N$ is

$$
V_{\tau}:=\{u \in M:(u, \tau) \in V\} .
$$

It will sometimes be convenient to abuse notation by not distinguishing explicitly between $V_{\tau}$ and $\pi_{2}^{-1}(\tau) \subset M \times N$. The fiber over $\tau$ is a cartesian product of subspheres of the spheres $M_{i}$ given by the equations defining $V$, so its topology is independent of $\tau$, and the following result is therefore quite unsurprising. In mathematical literature it is customary to assert a claim such as the following without proof, since to argue the point in detail would be a longwinded and mundane affair, and we shall follow suit ${ }^{(6)}$.

(6) It is, perhaps, worth mentioning that the "group" of the fibration may be taken to be the group $G$ defined below, and that a suitable atlas of coordinate functions (this terminology, and the definition of "fibration" we are appealing to, are from Steenrod (1951)) is given by maps of the following sort: given $\tau_{0} \in N$, a neighborhood $W \subset N$ of $\tau_{0}$, and a $C^{\infty}$ map $h: W \rightarrow G$ satisfying $h(\tau) \tau_{0}=\tau$ for all $\tau \in W$, let $\phi: V_{\tau_{0}} \times W \rightarrow \pi_{2}^{-1}(W)$ be given by $\phi(f, \tau):=(h(\tau) f, \tau)$ 
Proposition 7.2: $\pi_{2}: V \rightarrow N$ is a $C^{\infty}$ fibration.

Application of an integral formula (Shub and Smale (1993, p. 273), cf. Blum et. al. (1998, p. 240)) yields:

Proposition 7.3: For any open set $Z \subset V$,

$$
\int_{M} \#\left(\pi_{1}^{-1}(u) \cap Z\right) d u=\int_{N} \int_{V_{\tau} \cap Z} \operatorname{det}\left(A^{*}(u, \tau) A(u, \tau)\right)^{1 / 2} d u d \tau
$$

where $A(u, \tau): T_{\tau}(N) \rightarrow T_{u}(M)$ is the linear map whose graph is the orthogonal complement of $T V_{\tau}(u, \tau)$ in $T V(u, \tau)$ and $A^{*}(u, \tau)$ is the adjoint ${ }^{(7)}$ of $A(u, \tau)$.

The essential hypotheses implying this formula are that $M$ and $N$ are $C^{1}$ Riemannian manifolds, and $V$ is a $C^{1}$ submanifold of $M \times N$ with respect to which $\pi_{2}$ is a $C^{1}$ fibration. To obtain some intuition for this formula, consider the linear case which, as usual, is thought of as approximating the situation when $Z$ is "small." That is, let $\bar{M}$ and $\bar{N}$ be Euclidean spaces with $\operatorname{dim} \bar{M} \geq \operatorname{dim} \bar{N}$, let $\bar{V}$ is a linear subspace of $\bar{M} \times \bar{N}$ with $\operatorname{dim} \bar{V}=\operatorname{dim} \bar{M}$, let $\bar{\pi}_{1}$ and $\bar{\pi}_{2}$ be the restrictions of the projections, and assume that $\bar{\pi}_{2}$ is surjective. It is possible to pick coordinates for $\bar{M}$ with respect to which the formula is, by direct computation, a consequence of the change of variables formula. A proof for the linear case that does not refer to a coordinate system is given by Shub and Smale (1993). (See also Blum et. al. $(1998, \S 13.2)$.)

\subsection{Equivariant Group Actions}

We now show that the inner integral on the RHS of (5) does not depend on $\tau$ by using the symmetry that we built into the mathematical apparatus by displacing the space of mixed strategies for each agent from the unit simplex to the positive orthant of the unit sphere. We will be concerned with various actions of the group

$$
G:=O\left(\mathbb{R}^{T_{1}}\right) \times \ldots \times O\left(\mathbb{R}^{T_{n}}\right) .
$$

To begin with, there is the action of $G$ on $\mathbb{R}^{T_{1}} \times \ldots \times \mathbb{R}^{T_{n}}$ given by the rule

$$
g \tau:=\left(g_{1} \tau_{1}, \ldots, g_{n} \tau_{n}\right)
$$

Of course $N$ is an invariant of this action-that is, $g N=N$ for all $g \in G$-and there is an action of $G$ on $N$ given by restriction. We now describe a variety of actions of $G$ that are "complementary" to this action in the sense of the following result.

(7) Recall that if $X$ and $Y$ are inner product spaces, and $L: X \rightarrow Y$ is linear, the adjoint of $L$ is the linear transformation $A(L): Y \rightarrow X$ defined implicitly by the requirement that $\langle x, A(L) y\rangle=\langle L x, y\rangle$ for all $x \in X$ and $y \in Y$. 
Lemma 7.4: There is a unique action of $G$ on $\mathbb{R}^{T}$ with respect to which $\Theta$ is equivariant, and in which the action of each $g \in G$ on $\mathbb{R}^{T}$ is linear. In fact the action of each $g$ is an orthogonal transformation.

Proof: Identifying $t \in T$ with the correponding $n$-tuple of unit basis vectors in $\mathbb{R}^{T_{1}} \times \ldots \times$ $\mathbb{R}^{T_{n}},\{\Theta(t): t \in T\}$ is the standard basis for $\mathbb{R}^{T}$. For any $g \in G$ a linear transformation from $\mathbb{R}^{T}$ to itself (which we also denote by $g$ ) is determined by the condition $g(\Theta(t))=$ $\Theta(g t)$ for all $t \in T$, and since the functions $\tau \mapsto g(\Theta(\tau))$ and $\tau \mapsto \Theta(g \tau)$ are both multilinear and agree on a cartesian product of bases for the spaces $\mathbb{R}^{T_{i}}$, they must agree everywhere. For $g_{1}, g_{2} \in G$ and $t \in T$ we have

$$
\left(g_{1} g_{2}\right)(\Theta(t))=\Theta\left(g_{1} g_{2} t\right)=g_{1}\left(\Theta\left(g_{2} t\right)\right)=g_{1}\left(g_{2}(\Theta(t))\right),
$$

so we have defined a group action, and of course $\Theta$ is equivariant with respect to it.

To show that this is an action by orthogonal transformations we demonstrate that

$$
\left\langle\Theta(g \tau), \Theta\left(g \tau^{\prime}\right)\right\rangle=\left\langle\Theta(\tau), \Theta\left(\tau^{\prime}\right)\right\rangle
$$

for all $g \in G$ and $\tau, \tau^{\prime} \in \mathbb{R}^{T_{1}} \times \ldots \times \mathbb{R}^{T_{n}}$. When $g=\left(g_{1}, \ldots, g_{n}\right)$ has only one component $g_{i}$ differing from the identity we view $\mathbb{R}^{T}$ as a cartesian product of copies of $\mathbb{R}^{T_{i}}$ indexed by $T_{-i}$, so that $g_{i}$ acts on each copy in transforming $\Theta(\tau)$ to $\Theta(g \tau)$. The inner products on both sides are obtained by summing over the copies, so the claim follows from the fact that $g_{i}$ is an orthogonal transformation. The general case is obtained by applying this special case repeatedly.

The last result actually plays no role in subsequent events. It was included since its proof is simpler than the (omitted) proof of the following, by virtue of greater symmetry, even though the underlying ideas are the same.

Lemma 7.5: For each $i$ there is a unique action of $G$ on $\mathbb{R}^{T_{-i}}$ by orthogonal transformations with respect to which $\Theta_{i}$ is equivariant.

We think of $\mathbb{R}^{S_{i} \times T_{-i}}$ as a cartesian product of $\left|S_{i}\right|$ copies of $\mathbb{R}^{T_{-2}}$. For each $i$ and $s_{i} \in S_{i}$ let the function $\Theta_{i}^{s_{i}}: \mathbb{R}^{T_{1}} \times \ldots \times \mathbb{R}^{T_{n}} \rightarrow \mathbb{R}^{S_{i} \times T_{-i}}$ be given by $\Theta_{i}^{s_{i}}(\tau)=$ $\left(0, \ldots, 0, \Theta_{i}(\tau), 0, \ldots, 0\right)$ with $\Theta_{i}(\tau)$ the $s_{i}$-component. The action of $G$ in the next result is constructed by taking a cartesian product, indexed by the elements of $S_{i}$, of the action of $G$ given by the last result. Uniqueness follows from the uniqueness on each copy of $\mathbb{R}^{T=}$.

Lemma 7.6: For each $i$ there is a unique action of $G$ on $\mathbb{R}^{S_{i} \times T_{-i}}$ by orthogonal transformations such that, for all $s_{i} \in S_{i}, \Theta_{i}^{s_{i}}$ is equivariant.

In order to construct a suitable action on $\left(\mathbb{R}^{S}\right)^{*}$ we view a point $u_{i} \in\left(\mathbb{R}^{S}\right)^{*}$ as a pair

$$
\left(v_{i}, w_{i}\right) \in\left(\mathbb{R}^{S_{i} \times T_{-i}}\right)^{*} \times\left(\mathbb{R}^{S_{i} \times\left(S_{-i} \backslash T_{-i}\right)}\right)^{*},
$$

letting the action be given by the formula

$$
\begin{gathered}
g u_{i}=\left(g v_{i}, w_{i}\right)=\left(v_{i} \circ g^{-1}, w_{i}\right), \\
-30-
\end{gathered}
$$


where $g^{-1}$ on the RHS has the interpretation given by Lemma 7.6. Since $G$ acts on $\left(\mathbb{R}^{S_{i} \times T_{-i}}\right)^{*}$ by orthogonal transformations, the action on $\left(\mathbb{R}^{S}\right)^{*}$ is also by orthogonal transformations, so $M_{i}$ is an invariant, and usually we will focus on the action on this space given by restriction.

There is now an action of $G$ on $\left(\left(\mathbb{R}^{S}\right)^{*}\right)^{n}$ given by $g u=\left(g u_{1}, \ldots, g u_{n}\right)$, and an action on $\left(\left(\mathbb{R}^{S}\right)^{*}\right)^{n} \times N$ given by $g(u, \sigma)=(g u, g \sigma)$. Note the invariance of the payoffs resulting from deviations to the various pure strategies:

$$
\left\langle g v_{i}, \Theta_{i}^{s_{i}}(g \tau)\right\rangle=\left\langle v_{i}, \Theta_{i}^{s_{i}}(\tau)\right\rangle
$$

holds for all $g, \tau$, and $s_{i}$. Of course the main point of this construction is that $V$ and $\tilde{V}$ are invariants of this action: for all $g \in G$ we have

$$
g V=V \quad \text { and } \quad g \tilde{V}=\tilde{V} .
$$

For any $m$ the action of the orthogonal group $O\left(\mathbb{R}^{m+1}\right)$ on the unit sphere $S^{m}$ is transitive. As the cartesian product of transitive actions, the action of $G$ on $N$ is also transitive. To show that the inner integral in the formula above is independent of $\tau$ we apply the following invariances:

$$
\begin{array}{cl}
T_{g \tau}(N)=g\left(T_{\tau}(N)\right) ; & T_{g u}(M)=g\left(T_{u}(M)\right) ; \\
T_{(g u, g \tau)}\left(V_{g \tau}\right)=g\left(T_{(u, \tau)}\left(V_{\tau}\right)\right) ; & T_{(g u, g \tau)}(V)=g\left(T_{(u, \tau)}(V)\right) ; \\
\perp_{(g u, g \tau)}=g\left(\perp_{(u, \tau)}\right) ; \\
A(g u, g \tau)=g \circ A(u, \tau) \circ g^{-1} ; \quad A^{*}(g u, g \tau)=g \circ A^{*}(u, \tau) \circ g^{-1} .
\end{array}
$$

We present no proofs, since for most readers the underlying ideas are familiar, and at worst these are suitable exercises, since only straightforward linear algebra is involved.

If $Z \subset V$ is open and invariant under the action of $G$ (that is, $g Z=Z$ for all $g \in G)$ then the map $(u, \tau) \mapsto(g u, g \tau)$ is an isometry between $V_{\tau} \cap Z$ and $V_{g \tau} \cap Z$, so the change of variables formula, followed by application of the invariances above and elementary properties of the determinant, yields

$$
\int_{V_{\tau} \cap Z} \operatorname{det}\left(A^{*}(u, \tau) A(u, \tau)\right)^{1 / 2} d u=\int_{V_{g \tau} \cap Z} \operatorname{det}\left(A^{*}(u, g \tau) A(u, g \tau)\right)^{1 / 2} d u .
$$

Thus:

Lemma 7.7: For any open set $Z \subset V$ that is invariant under the action of $G$, any open $Y \subset N$, and any $\tau \in N$,

$$
\int_{M} \#\left(\pi_{1}^{-1}(u) \cap \pi_{2}^{-1}(Y) \cap Z\right) d u=\operatorname{vol}(Y) \cdot \int_{V_{\tau} \cap Z} \operatorname{det}\left(A^{*}(u, \tau) A(u, \tau)\right)^{1 / 2} d u .
$$




\subsection{Reduction to a Smaller Set of Variables}

The last result invites us to choose $\tau$ on the LHS however we please. The choice of $\tau=t^{0}=\left(t_{1}^{0}, \ldots, t_{n}^{0}\right)$ has the advantage that the integrand depends only on a few of the variables $u_{i}(s)$ defining $u$. For each $i$ let

$$
R_{-i}=\left\{t_{-i} \in T_{-i}: t_{j} \neq t_{j}^{0} \text { for exactly one } j\right\},
$$

and let $R_{i}=T_{i} \times R_{-i}$.

Proposition 7.8: Suppose that $u, u^{\prime} \in V_{t^{0}}$, and that, for each $i$, the restrictions of $u_{i}$ and $u_{i}^{\prime}$ to the subspace of $\mathbb{R}^{S}$ spanned by $R_{i}$ agree. Then $A\left(u, t^{0}\right)=A\left(u^{\prime}, t^{0}\right)$.

Proof: For $i=1, \ldots, n$ and $j=0, \ldots, p_{i}$, let $\Gamma_{i}^{j}: N \rightarrow \mathbb{R}^{S}$ be the function $\Gamma_{i}^{j}(\tau)=$ $\Theta\left(\tau \mid t_{i}^{j}\right)$. Fixing $u \in V_{t^{0}}$, a vector $(v, \psi) \in T_{\left(u, t^{0}\right)}(M \times N)$ is in the graph of $A\left(u, t^{0}\right)$ if and only if it is orthogonal to $T_{u}\left(V_{t^{0}}\right)$ and

$$
0=\left\langle u_{i},\left(D \Gamma_{i}^{j}\left(t^{0}\right)-D \Gamma_{i}^{0}\left(t^{0}\right)\right) \psi\right\rangle+\left\langle v_{i}, \Gamma_{i}^{j}\left(t^{0}\right)-\Gamma_{i}^{0}\left(t^{0}\right)\right\rangle
$$

for all $i$ and $j=1, \ldots, p_{i}$.

The fiber $V_{t^{0}}$ is a cartesian product, over $i$, of the subspheres of the spheres $M_{i}$ consisting of those $u_{i}$ whose kernels include the vectors $\left(t^{0} \mid t_{i}^{1}\right)-t^{0}, \ldots,\left(t^{0} \mid t_{i}^{p_{i}}\right)-t^{0}$. Consequently $(v, \psi)$ is orthogonal to $T_{u}\left(V_{t^{0}}\right)$ if and only if each $v^{i}$ is a linear combination of the vectors $\left(t^{0} \mid t_{i}^{1}\right)-t^{0}, \ldots,\left(t^{0} \mid t_{i}^{p_{i}}\right)-t^{0}$. We now see that $u_{i}$ affects $A\left(u, t^{0}\right)$ only through its restriction to the spaces $D \Gamma_{i}^{j}\left(t^{0}\right)\left(T_{t^{0}} N\right)$. The assertion follows from direct computation of the image of $D \Gamma_{i}^{j}\left(t^{0}\right)$.

The fiber $V_{t^{0}}$ is a cartesian product of the subsets of the spheres $M_{i}$ given by the conditions $u_{i}\left(t^{0}\right)=u_{i}\left(t^{0} \mid t_{i}^{1}\right)=\ldots=u_{i}\left(t^{0} \mid t_{i}^{p_{i}}\right)$, and, by the last result, the integrand depends only on certain other coordinates of $u$. The next step is to simplify by applying a change of variables that maps the subset of $M_{i}$ to a cartesian product, with one factor being the space of the irrelevant variables, whose effect on the integral reduces to multiplication by the volume of this space. Although the next two lemmas are notationally cumbersome, the underlying idea is simple. The complex appearance is due entirely to the complexity of the intended application's environment.

Lemma 7.9: For $i=1, \ldots, n$ suppose that:

(a) $a_{i}, b_{i}, c_{i}$ are nonnegative integers;

(b) $D^{i}$ is the unit disk in $\mathbb{R}^{a_{i}}$;

(c) $E^{i}$ is the subsphere of the unit sphere in $\mathbb{R}^{b_{i}+c_{i}}$ consisting of those unit vectors $\left(q^{i}, r^{i}\right)$ such that $q_{1}^{i}=\ldots=q_{b_{i}}^{i}$

(d) $F^{i}$ is the subsphere of the unit sphere in $\mathbb{R}^{a_{i}+b_{i}+c_{i}}$ consisting of those unit vectors $\left(p^{i}, q^{i}, r^{i}\right)$ such that $q_{1}^{i}=\ldots=q_{b_{i}}^{i}$.

For each $i$ define $g^{i}: D^{i} \times E^{i} \rightarrow F^{i}$ by

$$
g^{i}\left(p^{i},\left(q^{i}, r^{i}\right)\right)=\left(p^{i},\left(1-\left\|p^{i}\right\|^{2}\right)^{1 / 2} q^{i},\left(1-\left\|p^{i}\right\|^{2}\right)^{1 / 2} r^{i}\right) .
$$


Let

$$
F=\left\{(p, q, r) \in \prod_{i} \mathbb{R}^{a_{i}} \times \prod_{i} \mathbb{R}^{b_{i}} \times \prod_{i} \mathbb{R}^{c_{i}}:\left(p^{i}, q^{i}, r^{i}\right) \in F^{i} \text { for all } i=1, \ldots, n .\right\} .
$$

and let $g: D \times E \rightarrow F$ be the function which takes $(p,(q, r))$ to the point $(\hat{p}, \hat{q}, \hat{r}) \in F$ such that, for each $i,\left(\hat{p}^{i}, \hat{q}^{i}, \hat{r}^{i}\right)=g^{i}\left(p^{i},\left(q^{i}, r^{i}\right)\right)$. Then for all $(p, q, r) \in D \times E$,

$$
|\operatorname{det} D g(p,(q, r))|=\prod_{i=1}^{n}\left(1-\left\|p^{i}\right\|^{2}\right)^{\frac{c_{i}-1}{2}} \text {. }
$$

Proof: First we compute that

$$
\left\|D g^{i}\left(p^{i},\left(q^{i}, r^{i}\right)\right) \frac{p^{i}}{\left\|p^{i}\right\|}\right\|=\left\|\left.\frac{d\left(x,\left(1-x^{2}\right)^{1 / 2}\right)}{d x}\right|_{x=\left\|p^{i}\right\|}\right\|=\left(1-\left\|p^{i}\right\|^{2}\right)^{-1 / 2} .
$$

Insofar as $\operatorname{dim} E=c_{i}$, if we compute with respect to orthonormal bases for $T_{p} D$ and $T_{(q, r)} E$, where the first basis includes $p^{i} /\left\|p^{i}\right\|$, we find that

$$
\left|\operatorname{det} D g^{i}\left(p^{i},\left(q^{i}, r^{i}\right)\right)\right|=\left(1-\left\|p^{i}\right\|^{2}\right)^{\frac{c_{i}-1}{2}} .
$$

With respect to such bases, the matrix of $D g$ is block diagonal with the matrices of the $D g^{i}$ as the blocks, so that

$$
|\operatorname{det} D g(p,(q, r))|=\left|\operatorname{det} D g^{1}\left(p^{1},\left(q^{1}, r^{1}\right)\right)\right| \cdot \ldots \cdot\left|\operatorname{det} D g^{n}\left(p^{n},\left(q^{n}, r^{n}\right)\right)\right| \text {. }
$$

Lemma 7.10: In addition to the hypotheses of the last result, suppose that. for each $i=1, \ldots n, \tilde{E}^{i}$ is an open subset of $E^{i}$, and let

$$
\tilde{F}^{i}=\left\{\left(p^{i}, q^{i}, r^{i}\right) \in F^{i}:\left(q^{i}, r^{i}\right) \neq(0,0) \text { and } \frac{\left(q^{i}, r^{i}\right)}{\left\|\left(q^{i}, r^{i}\right)\right\|} \in \tilde{E}^{i}\right\} .
$$

Set

$$
\tilde{E}=\left\{(q, r) \in E:\left(q^{i}, r^{i}\right) \in \tilde{E}^{i} \text { for all } i=1, \ldots, n .\right\}
$$

and

$$
\tilde{F}=\left\{(p, q, r) \in F:\left(p^{i}, q^{i}, r^{i}\right) \in \tilde{F}^{i} \text { for all } i=1, \ldots, n .\right\} .
$$

If $h: D \rightarrow \mathbb{R}$ is a bounded measurable function, then

$$
\int_{\tilde{F}} h(p) d(p, q, r)=\operatorname{vol}(\tilde{E}) \cdot \int_{D} h(p)\left(\prod_{i=1}^{n}\left(1-\left\|p^{i}\right\|^{2}\right)^{\frac{c_{1}-1}{2}}\right) d p .
$$

Proof: Applying the last result, the change of variables formula gives

$$
\int_{\tilde{F}} h(p) d(p, q, r)=\int_{D \times \tilde{E}} h(p)\left(\prod_{i=1}^{n}\left(1-\left\|p^{i}\right\|^{2}\right)^{\frac{c_{j}-1}{2}}\right) d p
$$

and the asserted equation follows from an application of Fubini's theorem. 


\subsection{Completing the Proof}

For any open $Y \subset N$ Lemma 7.7 implies that

$$
\nu(Y)=\operatorname{vol}(Y) \cdot \int_{V_{\tau}} \operatorname{det}\left(A^{*}(u, \tau) A(u, \tau)\right)^{1 / 2} d u
$$

and

$$
\tilde{\nu}(Y)=\operatorname{vol}(Y) \cdot \int_{V_{\tau} \cap \tilde{V}} \operatorname{det}\left(A^{*}(u, \tau) A(u, \tau)\right)^{1 / 2} d u .
$$

In the applications of Lemma $7.10, D^{i}$ is the unit disk in $\mathbb{R}^{R_{i}}$, and $E^{i}$ is the set of elements of the unit sphere in $\mathbb{R}^{S \backslash R^{i}}$ with

$$
u_{i}\left(t^{0}\right)=u_{i}\left(t^{0} \mid t_{i}^{1}\right)=\ldots=u_{i}\left(t^{0} \mid t_{i}^{p_{i}}\right) .
$$

We apply Lemma 7.10 twice, once with $\tilde{E}^{i}=E^{i}$ and once with

$$
\tilde{E}^{i}:=\left\{u_{i} \in E^{i}: u_{i}\left(t^{0}\right)>u_{i}\left(t^{0} \mid s_{i}\right) \text { for all } s_{i} \in S_{i} \backslash T_{i}\right\},
$$

after which, by taking the ratio of the two formulas, we obtain

$$
\frac{\tilde{\nu}(Y)}{\nu(Y)}=\frac{\operatorname{vol}(\tilde{E})}{\operatorname{vol}(E)}=\prod_{i=1}^{n} \frac{\operatorname{vol}\left(\tilde{E}^{i}\right)}{\operatorname{vol}\left(E^{i}\right)}
$$

Now Proposition 2.2 follows from Lemma A.2 in Appendix A.

\section{The Derived Density on the Simplex}

We have seen that the distribution of mixed strategy vectors induced by Nash equilibrium is uniform when mixed strategy vectors are parameterized to lie in $N^{++}$. In this section we study the corresponding distribution on the traditional parameterization of the space of mixed strategy vectors, the cartesian product of simplices. The uniform distribution on $\mathrm{N}^{++}$is the product of the uniform distributions on the spaces $N_{i}^{++}$. Each of these factors induces a measure on the unit simplex in $\mathbb{R}^{T_{2}}$ via radial projection, and the induced distribution on the cartesian product of the simplices is the product of these induced measures. We aim at characterizing the induced measure on the unit simplex in $\mathbb{R}^{T_{2}}$.

To simplify the exposition, and avoid repeated references to an arbitrary agent, we adopt a new system of notation. Fix an integer $m \geq 1$. Let $\mathbf{b}=\left(\frac{1}{m}, \ldots, \frac{1}{m}\right)$ be the barycenter of the unit simplex, and let

$$
\Delta:=\left\{d \in \mathbb{R}^{m}: d \cdot \mathbf{b}=\frac{1}{m}\right\}
$$

be the affine $(m-1)$-plane that contains the unit simplex. Let $\mathbf{e}:=\sqrt{m} \mathbf{b}$ be the unit vector parallel to $\mathrm{b}$, and let

$$
\Sigma:=\left\{p \in S^{m-1}: p \cdot \mathbf{e}>0\right\}
$$


be the 'Northern hemisphere' defined by the 'North pole' $\sqrt{m} \mathbf{b}$. Let $\rho: \Sigma \rightarrow \Delta$ be the radial projection of $\Sigma$ onto $\Delta$ :

$$
\rho(p):=\frac{1}{m(p \cdot \mathbf{b})} p .
$$

Let $\Sigma^{++}$and $\Delta^{++}$be the intersections of $\Sigma$ and $\Delta$ with the interior of the positive orthant of $\mathbb{R}^{m}$. Of course $\rho\left(\Sigma^{++}\right)=\Delta^{++}$. The induced measure on $\Delta^{++}$is

$$
\beta:=\mathbf{U}_{\Sigma^{++}} \circ\left(\rho \mid \Sigma^{++}\right)^{-1} .
$$

Let $f: \Delta^{++} \rightarrow(0, \infty)$ be the density of $\beta$ with respect to $\mathbf{U}_{\Delta^{++}}$.

Eventually we will give a closed form formula for this function. The first step in the calculation is to compute the value of $f$ at $\mathbf{b}$, which we do by computing the ratio of the measures assigned to disks of small radius around $\mathbf{b}$ by $\beta$ and $\mathbf{U}_{\Delta^{++}}$. For $r \geq 0$ let

$$
\mathbf{B}(r)=\{\delta \in \Delta:\|\delta-\mathbf{b}\|<r\}
$$

Note that $\mathbf{B}(r) \subset \Delta^{++}$when

$$
r<\left\|\left(\frac{1}{m+1}, \ldots, \frac{1}{m+1}, 0\right)-\mathbf{b}\right\|=\frac{1}{\sqrt{(m-1) m}} .
$$

It is very well known that

$$
\operatorname{vol}(\mathbf{B}(r))=\frac{\Gamma\left(\frac{1}{2}\right)^{m-1}}{\Gamma\left(\frac{m+1}{2}\right)} r^{m-1} .
$$

It is also well known that $\operatorname{vol}\left(\Delta^{++}\right)=\frac{\sqrt{m}}{m !}$. (One way to compute this is to observe that $\left\{x \in \mathbb{R}_{>0}^{m}: x_{1}+\ldots+x_{m}<1\right\}$, whose volume is $1 / m$ !, is a pyramid with base $\Delta^{++}$and the origin as the apex.) Therefore

$$
\mathbf{U}_{\Delta^{++}}(\mathbf{B}(r))=\frac{m ! \Gamma\left(\frac{1}{2}\right)^{m-1}}{\sqrt{m} \Gamma\left(\frac{m+1}{2}\right)} r^{m-1}
$$

when $0<r<\frac{1}{\sqrt{(m-1) m}}$.

- The set in $\Sigma$ corresponding to $\mathbf{B}(r)$ is $\rho^{-1}(\mathbf{B}(r))=\{\sigma \in \Sigma: \angle(\sigma, \mathbf{e})<\theta(r)\}$ where $\theta(r):=\arctan \frac{r}{\|\mathbf{b}\|}$. The set in $S^{m-1}$ consisting of those points $y$ with $\angle(\mathbf{e}, y)=\alpha$ is a sphere of radius $\sin \alpha$, so

$$
\operatorname{vol}\left(\rho^{-1}(\mathbf{B}(r))\right)=\operatorname{vol}\left(S^{m-2}\right) \cdot \int_{0}^{\theta(r)}(\sin \alpha)^{m-2} d \alpha=2 \frac{\Gamma\left(\frac{1}{2}\right)^{m-1}}{\Gamma\left(\frac{m-1}{2}\right)} \cdot \int_{0}^{\theta(r)}(\sin \alpha)^{m-2} d \alpha
$$

and

$$
\operatorname{vol}\left(\Sigma^{++}\right)=2^{-m} \operatorname{vol}\left(S^{m-1}\right)=\frac{\Gamma\left(\frac{1}{2}\right)^{m}}{2^{m-1} \Gamma\left(\frac{m}{2}\right)} .
$$


Therefore

$$
\beta(\mathbf{B}(r))=\frac{2^{m} \Gamma\left(\frac{m}{2}\right)}{\Gamma\left(\frac{1}{2}\right) \Gamma\left(\frac{m-1}{2}\right)} \cdot \int_{0}^{\theta(r)}(\sin \alpha)^{m-2} d \alpha .
$$

Combining this with the formula above, and noting that $\Gamma\left(\frac{m+1}{2}\right)=\frac{m-1}{2} \Gamma\left(\frac{m-1}{2}\right)$, yields:

\section{Lemma 8.1:}

$$
\frac{\beta(\mathbf{B}(r))}{\mathbf{U}_{\Delta^{++}}(\mathbf{B}(r))}=\frac{\sqrt{m}(m-1) 2^{m-1} \Gamma\left(\frac{m}{2}\right)}{m ! \Gamma\left(\frac{1}{2}\right)^{m}} \cdot \frac{\int_{0}^{\theta(r)}(\sin \alpha)^{m-2} d \alpha}{r^{m-1}} .
$$

Since $\frac{\sin \alpha}{\alpha}$ is a decreasing function of $\alpha$,

$$
\begin{aligned}
\frac{(\sin \theta(r))^{m-2} \theta(r)}{m-1}= & \left(\frac{\sin \theta(r)}{\theta(r)}\right)^{m-2} \int_{0}^{\theta(r)} \alpha^{m-2} d \alpha<\int_{0}^{\theta(r)}(\sin \alpha)^{m-2} d \alpha \\
& <\int_{0}^{\theta(r)} \alpha^{m-2} d \alpha=\frac{\theta(r)^{m-1}}{m-1} .
\end{aligned}
$$

Since $\lim _{r \rightarrow 0} \theta(r) / r=\sqrt{m}$,

$$
\lim _{r \rightarrow 0} \frac{\int_{0}^{\theta(r)}(\sin \alpha)^{m-2} d \alpha}{r^{m-1}}=\frac{\sqrt{m^{m-1}}}{m-1}
$$

and by taking the limit as $r \rightarrow 0$ of the RHS of the formula above, then substituting $\Gamma\left(\frac{1}{2}\right)=\sqrt{\pi}$, we obtain:

Lemma 8.2: $f(\mathbf{b})=\frac{1}{2}\left(2 \sqrt{\frac{m}{\pi}}\right)^{m} \frac{\Gamma\left(\frac{m}{2}\right)}{m !}$.

We now compute the ratio $f(p) / f(\mathbf{b})$ for an arbitrary point $p \in \Delta^{++}$.

Lemma 8.3: For $q \in \Sigma$, $\operatorname{det}[D \rho(q)]=\frac{\sqrt{m}}{(\sqrt{m} \cos L(q, \mathbf{e}))^{m}}$.

Proof: The formula defining $\rho$ is meaningful for any $q$ with $q \cdot \mathbf{e}>0$, and we will abuse notation by letting $\rho$ also denote the extension to this domain. From the defining formula for $\rho$ it is easy to compute directly that

$$
D \rho(q) \mathbf{e}=\frac{1}{\sqrt{m}(q \cdot \mathbf{e})}(\mathbf{e}-q /(q \cdot \mathbf{e})),
$$

while if $v \in \mathbb{R}^{m}$ is orthogonal to $\mathbf{e}$, then

$$
D \rho(q) v=\frac{1}{\sqrt{m}(q \cdot \mathbf{e})} v .
$$

In computing the determinant of the restriction of $D \rho(q)$ to $T_{q} \Sigma$ we will work with an orthonormal basis $\mathbf{e}_{1}, \ldots, \mathbf{e}_{m}$ of $\mathbb{R}^{m}$ in which $\mathbf{e}_{1}=\mathbf{e}$ and $q$ is spanned by $\mathbf{e}_{1}$ and $\mathbf{e}_{2}$, so 
that $q=(\cos \theta) \mathbf{e}_{1}+(\sin \theta) \mathbf{e}_{2}$ for some $\theta$. Note that $(-\sin \theta) \mathbf{e}_{1}+(\cos \theta) \mathbf{e}_{2}, \mathbf{e}_{3}, \ldots, \mathbf{e}_{m}$ is an orthonormal basis of $T_{q} \Sigma$, while $\mathbf{e}_{2}, \ldots, \mathbf{e}_{m}$ is an orthonormal basis for $T_{\rho(q)} \Delta$.

Since

$$
\mathbf{e}_{1}-q /\left(q \cdot \mathbf{e}_{1}\right)=\mathbf{e}_{1}-\frac{(\cos \theta) \mathbf{e}_{1}+(\sin \theta) \mathbf{e}_{2}}{\cos \theta}=-\frac{\sin \theta}{\cos \theta} \mathbf{e}_{2}
$$

we may compute that

$$
\begin{aligned}
D \rho(q)\left((\cos \theta) \mathbf{e}_{1}+\right. & \left.(\sin \theta) \mathbf{e}_{2}\right)=(-\sin \theta) D \rho(q) \mathbf{e}_{1}+(\cos \theta) D \rho(q) \mathbf{e}_{2} \\
& =\frac{1}{\sqrt{m}\left(q \cdot \mathbf{e}_{1}\right)}\left((-\sin \theta)\left(\mathbf{e}_{1}-q /\left(q \cdot \mathbf{e}_{1}\right)\right)+(\cos \theta) \mathbf{e}_{2}\right) \\
& =\frac{1}{\sqrt{m} \cos \theta}\left(\frac{1}{\cos \theta} \mathbf{e}_{2}\right) .
\end{aligned}
$$

For $j=3 \ldots, m$ we have

$$
D \rho(q) \mathbf{e}_{j}=\frac{1}{\sqrt{m}(q \cdot \mathbf{e})} \mathbf{e}_{j}=\frac{1}{\sqrt{m} \cos \theta} \mathbf{e}_{j}
$$

Therefore, with respect to the indicated bases, the matrix of $D \rho(q)$ is diagonal, and, as asserted,

$$
\operatorname{det}[D \rho(q)]=\frac{1}{\sqrt{m} \cos ^{2} \theta}\left(\frac{1}{\sqrt{m} \cos \theta}\right)^{m-2}
$$

By the change of variables formula, for $p \in \Delta^{++}$the density $f(p)$ is inversely proportional to $\operatorname{det}\left[D \rho\left(\rho^{-1}(p)\right)\right]$, so, taking the ratio of this quantity at $p$ and at $\mathbf{b}$, then multiplying by the density $\mathbf{a t} \mathbf{b}$ as given by Lemma 8.2 , we obtain

Theorem 3: For $p \in \Delta^{++}$,

$$
f(p)=\frac{1}{2}\left(2 \sqrt{\frac{m}{\pi}} \cos \angle(p, \mathbf{e})\right)^{m} \frac{\Gamma\left(\frac{m}{2}\right)}{m !}
$$

The main conceptual point we wish to establish is that, in comparison with $\mathbf{U}_{\Delta^{++}}$, $\beta$ is sharply concentrated near the barycenter of $\Delta^{++}$. Recall Stirling's formula (e.g., Bitlingsley $(1986,18.21))$ :

$$
m !=K_{m} \cdot \sqrt{2 \pi} \frac{m^{m+\frac{1}{2}}}{e^{m}} \quad \text { where } 1<K_{m}<\sqrt{1+\frac{1}{m}} .
$$

Also recall the basic properties of the function $\Gamma(\cdot): \Gamma\left(\frac{1}{2}\right)=\sqrt{\pi}, \Gamma(1)=1$, and $s \Gamma(s)=$ $\Gamma(s+1)$ for $0<s<\infty$. In the event that $m$ is even we have

$$
\Gamma\left(\frac{m}{2}\right)=\left(\frac{m}{2}-1\right) !=\frac{(m / 2) !}{m / 2}
$$


and after some simplifications, the result of applying this to the RHS in Lemma 8.2 is

$$
f(\mathbf{b})=\frac{K_{\frac{m}{2}}}{K_{m}} \cdot \frac{1}{\sqrt{2} m}\left(\frac{2 e}{\pi}\right)^{\frac{m}{2}} \text {. }
$$

When $m$ is odd, we have

$$
\begin{aligned}
\Gamma\left(\frac{m}{2}\right) & =\Gamma\left(\frac{1}{2}\right) \cdot \frac{1}{2} \cdot \frac{3}{2} \cdot \ldots \cdot \frac{m-2}{2} \\
& =\Gamma\left(\frac{1}{2}\right) \cdot \frac{1}{2} \cdot \frac{2}{2} \cdot \frac{3}{2} \cdot \ldots \cdot \frac{m-3}{m-3} \cdot \frac{m-2}{2} \cdot \frac{m-1}{m-1}=\frac{\Gamma\left(\frac{1}{2}\right)(m-1) !}{2^{m-1}\left(\frac{m-1}{2}\right) !} .
\end{aligned}
$$

In this event a little calculation shows that

$$
f(\mathbf{b})=\frac{1}{K_{\frac{m-1}{2}}} \cdot \frac{1}{\sqrt{2 e} m}\left(\frac{m}{m-1}\right)^{\frac{m}{2}} \cdot\left(\frac{2 e}{\pi}\right)^{\frac{m}{2}} .
$$

In both cases the density at the barycenter grows exponentially with $m$.

Other senses in which $\beta$ is concentrated near the barycenter flow out of the computation of the density at particular points. For $k=1, \ldots, m$ let

$$
b_{k}=\rho^{-1}\left(\frac{1}{k}, \ldots, \frac{1}{k}, 0, \ldots, 0\right)=\left(\frac{1}{\sqrt{k}}, \ldots, \frac{1}{\sqrt{k}}, 0, \ldots, 0\right)
$$

be the barycenter of $(k-1)$-dimensional face of the $(k-1)$-dimensional simplex spanned by the first $k$ standard unit basis vectors. The message that emerges most forcefully from the following result is that $\beta$ assigns very little mass to the parts of the simplex near the vertices.

Proposition 8.4: For $k=1, \ldots, m$,

$$
\operatorname{det}\left[D \rho\left(b_{k}\right)\right]=\sqrt{\frac{m}{k^{m}}} \quad \text { and } \quad \frac{\operatorname{det}\left[D \rho\left(b_{k}\right)\right]}{\operatorname{det}\left[D \rho\left(b_{m}\right)\right]}=\left(\frac{m}{k}\right)^{\frac{m}{2}}
$$

Proof: This follows immediately from the last result once we compute that

$$
\cos \angle\left(b_{k}, \mathbf{e}\right)=b_{k} \cdot b_{m}=k \cdot \frac{1}{\sqrt{k}} \cdot \frac{1}{\sqrt{m}}=\sqrt{\frac{k}{m}} .
$$

Perhaps the most balanced assessment of the extent to which $\beta$ is concentrated near the barycenter results from studying the measure of $\mathbf{B}\left(\sqrt{\frac{1}{(m-1) m}}\right)$. On the one hand $\beta$ assigns much greater measure to this set than does $\mathbf{U}_{\Delta^{++}}$:

$$
\frac{\beta\left(\mathbf{B}\left(\sqrt{\frac{1}{(m-1) m}}\right)\right)}{\mathbf{U}_{\Delta^{+}+}\left(\mathbf{B}\left(\sqrt{\frac{1}{(m-1) m}}\right)\right)}>f\left(\mathbf{b}_{m-1}\right) \approx\left(\frac{m-1}{m}\right)^{\frac{m}{2}} \frac{1}{\sqrt{2} m}\left(\frac{2 e}{\pi}\right)^{\frac{m}{2}} \approx \frac{1}{\sqrt{2 e} m}\left(\frac{2 e}{\pi}\right)^{\frac{m}{2}}
$$


On the other hand, we may apply Stirling's formula to the equation for $\beta\left(\mathbf{B}\left(\sqrt{\frac{1}{(m-1) m}}\right)\right)$, finding, after a bit of computation, that in the case of odd $m$ (the case of even $m$ is similar) the total $\beta$-measure of this ball is not large:

$$
\begin{aligned}
\beta\left(\mathbf{B}\left(\sqrt{\frac{1}{(m-1) m}}\right)\right) & =\frac{K_{m}}{K_{\frac{m-1}{2}}} \sqrt{\frac{(m-1) m}{\pi}}\left(\frac{m}{m-1}\right)^{\frac{m}{2}}\left(\frac{2 \pi}{m-1}\right)^{\frac{m}{2}} \\
& \approx \sqrt{\frac{(m-1) m e}{\pi}}\left(\frac{2 \pi}{m-1}\right)^{\frac{m}{2}}
\end{aligned}
$$




\section{References}

1. D. N. Bernstein, The number of roots of a system of equations, Functional Analysis and its Applications 9 (1975), 183-185.

2. P. Billingsley, Probability and Measure, (2nd ed.), Wiley, New York, (1986).

3. L. Blum, F. Cucker, M. Shub, and S. Smale, Complexity and Real Computation, SpringerVerlag, New York, (1998).

4. M. Dresher, Probability of a pure equilibrium point in $n$-person games, Journal of Combinatorial Theory 8 (1970), 134-145.

5. A. Edelman and E. Kostlan, How many zeros of a random polynomial are real?, Bulletin of the American Mathematical Society 32 (1995), 1-37.

6. G. P. Egorychev, Van der Waerden conjecture and applications, Handbook of Algebra, Vol. I, Elsevier, Amsterdam (1996), 3-26.

7. G. Ewald, Combinatorial Convexity and Algebraic Geometry, Springer-Verlag. New York, (1996).

8. H. Federer, Geometric Measure Theory, Springer, New York, (1969).

9. D. Fudenberg and D. K. Levine, The Theory of Learning in Games, MIT Press, Cambridge, MA (1998).

10. V. Guillemin and A. Pollack, Differential Topology, Prentice-Hall, Englewood Cliffs, (1965).

11. F. Gul, D. Pearce, and E. Stachetti, A bound on the proportion of pure strategy equilibria in generic games, Mathematics of Operations Research 18 (1993), 548- 552.

12. J. C. Harsanyi, Oddness of the number of equilibrium points: a new proof, Int. J. Game Theory. 2 (1973), 235-250.

13. J. C. Harsanyi and R. Selten, A General Theory of Equilibrium Selection in Games, MIT Press, Cambridge, MA (1988).

14. M. Kac, On the average number of real roots of a random algebraic equation. Bulletin of the American Mathematical Society 49 (1943), 314-320 and 938.

15. A. G. Khovanskii, Newton polyhedra and the genus of complete intersections, Functional Analysis and its Applications 12 (1978), 51-61.

16. E. Kostlan, On the distribution of roots of random polynomials. From Topology to Computation: Proceedings of the Smalefest, Hirsch, M., Marsden, J., and Shub, M. (eds) (1993).

17. D. M. Kreps, Game Theory and Economic Modelling, Oxford:Clarendon Press, New York, (1990).

18. A. G. Kushnirenko, The Newton polyhedron and the number of solution of a system of $k$ equations in $k$ unknowns, Upsekhi Mat. Nauk. 30 (1975), 266-267.

19. R. D. Luce and H. Raiffa, Games and Decisions; Introduction and Critical Survey, Wiley, New York, (1957).

20. R. D. McKelvey and A. McLennan, The maximal number of regular totally mixed Nash equilibria, Journal of Economic Theory 72 (1997), 411-425.

21. A. McLennan, The maximal generic number of pure Nash equilibria, Journal of Economic Theory 72 (1997a), 408-410.

22. A. McLennan, The maximal number of real roots of a multihomogeneous system of polynomial equations, forthcoming in Beiträge zur Algebra und Geometrie, (1998a).

23. A. McLennan, The maximal number of real roots of a multihomogeneous system of polynomial equations, mimeo, University of Minnesota, (1998b). 
24. L. Nachbin, The Haar Integral, Van Nostrand, New York (1965).

25. I. Powers, Limiting distributions of the number of pure strategy Nash equilibria, International Journal of Game Theory 19 (1990), 277-286.

26. J. M. Rojas, On the average number of real roots of certain random sparse polynomial systems, Lectures on Applied Mathematics Series, ed. by J. Renegar, M. Shub, and S. Smale, American Mathematical Society, 1996.

27. M. Shub and S. Smale, Complexity of Bezout's theorem II: volumes and probabilities, Computational Algebraic Geometry (F. Eyssette and A. Galligo, eds.), Progr. Math., vol. 109 (1993), Birkhauser, Boston, 267-285.

28. S. Smale, On the average number of steps of the simplex method of linear programming, Mathematical Programming 27 (1983), 241-262.

29. W. Stanford, The Poisson distribution and probability of $k$ pure equilibria in matrix games, mimeo, Department of Economics, University of Illinois at Chicago, (1993). [UPDATE]

30. N. Steenrod, The Topology of Fibre Bundles, Princeton University Press, Princeton, (1951).

31. E. van Damme, Stability and Perfection of Nash Equilibria, Springer-Verlag, New York, (1987).

32. J. W. Weibull, Evolutionary Game Theory, MIT Press, Cambridge, MA (1995). 


\section{Appendix A. The Function $r(a, b)$}

This appendix gathers together various bits of information about the function $r$. Recall that, for $a, b \geq 0, r(a, b)$ was defined in Section 2 to be the probability that

$$
\frac{\epsilon_{0}}{\sqrt{a+1}}>\max \left\{\epsilon_{1}, \ldots, \epsilon_{b}\right\}
$$

where $\epsilon_{0}, \epsilon_{1}, \ldots \epsilon_{b}$ are i.i.d. normal random variables. Our results consist of two equivalent characterizations of $r$, some elementary facts, two lower bounds, and numerical results giving a sense of the quality of these bounds.

Let $\Phi$ be the cumulative distribution for a variable $\epsilon$ that is distributed normally with mean 0 and unit variance. Integration, over possible realizations of $\epsilon_{0}$, of the probability. conditional on $\epsilon_{0}$, that $\frac{\epsilon_{0}}{\sqrt{a+1}}>\max \left\{\epsilon_{1}, \ldots, \epsilon_{b}\right\}$, yields:

Lemma A.1:

$$
r(a, b)=\frac{1}{\sqrt{2 \pi}} \int_{-\infty}^{\infty} \Phi\left(\frac{s}{\sqrt{a+1}}\right)^{b} e^{-s^{2} / 2} d s .
$$

Using numerical integration, for particular values of $a$ and $b$ it is possible to evaluate this expression with great accuracy.

The following geometric interpretation of $r$ was used in Section 8.

Lemma A.2: For nonnegative integers $a, b, c$ with $a, b \geq 0$, and $c \geq a+b$,

$$
r(a, b)=\frac{\operatorname{vol}\left(\left\{x \in S^{c}: x_{0}=x_{1}=\ldots=x_{a}>\max \left\{x_{a+1}, \ldots, x_{a+b}\right\}\right\}\right)}{\operatorname{vol}\left(\left\{x \in S^{c}: x_{0}=x_{1}=\ldots=x_{a}\right\}\right)} .
$$

Proof: Let $\mathbf{e}_{0}, \mathbf{e}_{1}, \ldots, \mathbf{e}_{c}$ be the unit basis vectors of $\mathbb{R}^{c+1}$. Let $\epsilon_{0}, \epsilon_{a+1}, \epsilon_{a+2}, \ldots, \epsilon_{c}$ be i.i.d. normal random variables, let

$$
\tilde{x}=\epsilon_{0} \cdot \frac{\mathbf{e}_{0}+\mathbf{e}_{1}+\ldots+\mathbf{e}_{a}}{\sqrt{a+1}}+\epsilon_{a+1} \cdot \mathbf{e}_{a+1}+\ldots+\epsilon_{c} \cdot \mathbf{e}_{c},
$$

and let $\tilde{y}=\tilde{x} /\|\tilde{x}\|$. By virtue of standard properties of the multivariate normal distribution, $\tilde{y}$ is a uniformly distributed random point in the sphere $\left\{x \in S^{c}: x_{0}=x_{1}=\ldots=\right.$ $\left.x_{a}\right\}$. The ratio of volumes in the assertion is now seen to be the probability of the event $\epsilon_{0} / \sqrt{a+1}>\max \left\{\epsilon_{a+1}, \ldots \epsilon_{a+b}\right\}$.

There are several cases in which $r(a, b)$ can be characterized exactly.

Lemma A.3: For any $a, r(a, 0)=1$ and $r(a, 1)=1 / 2$.

Proof: The first assertion is obvious. To prove the second observe that, for i.i.d. normal random variables $\epsilon_{0}, \epsilon_{1}$, the pair $\left(\frac{1}{\sqrt{a+1}} \epsilon_{0}, \epsilon_{1}\right)$ is distributed symmetrically with respect to negation, in the sense that, for any $(x, y) \in \mathbb{R}^{2}$, the densities at the points $(x, y)$ and $(-x,-y)$ are equal. 
Lemma A.4: For any $b, r(0, b)=\frac{1}{b+1}$.

Proof: Each of the i.i.d. normal random variables $\epsilon_{0}, \epsilon_{1}, \ldots, \epsilon_{b}$ is equally likely to be maximal.

We now present two lower bounds on $r(a, b)$, each of which may be useful in asymptotic analysis. For $a, b \geq 0$ let

$$
p(a, b)=\frac{2^{-(b+1)}}{b+1} \cdot\left(1+\sqrt{a+1}\left[\left(1+\frac{1}{\sqrt{a+1}}\right)^{b+1}-1\right]\right)
$$

and

$$
q(a, b)=\left(\begin{array}{c}
a+b+1 \\
a+1
\end{array}\right)^{-1}=\frac{(a+1) ! b !}{(a+b+1) !}
$$

Lemma A.5: $r(a, b) \geq p(a, b)$.

Proof: We partition the space of realizations of $\epsilon_{0}, \epsilon_{1}, \ldots, \epsilon_{b}$ according to the signs of these variables. There are $2^{b+1}$ equiprobable cells in this partition in which no variable vanishes. To begin with note that

$$
\operatorname{Pr}\left(0>\frac{\epsilon_{0}}{\sqrt{a+1}}>\max \left\{\epsilon_{1}, \ldots, \epsilon_{b}\right\}\right) \geq \operatorname{Pr}\left(0>\epsilon_{0}>\max \left\{\epsilon_{1}, \ldots, \epsilon_{b}\right\}\right)=\frac{2^{-(b+1)}}{b+1}
$$

In all other cells $\epsilon_{j}>0$ for some $j \geq 1$, and we must have $\epsilon_{0}>0$ in a cell in order for the cell to make any contribution to the probability that $\frac{\epsilon_{0}}{\sqrt{a+1}}>\max \left\{\epsilon_{1}, \ldots, \epsilon_{b}\right\}$. On the interval $[0, \infty)$ the density of the normal distribution is monotonic decreasing. Integrating over the possible realizations of $\epsilon_{0}$, we find that, conditional on $\epsilon_{0}, \epsilon_{j}>0$, the probability that $\epsilon_{j}<\epsilon_{0} / \sqrt{a+1}$ is greater than $1 / \sqrt{a+1}$ times that probability that $\epsilon_{j}<\epsilon_{0}$. More generally,

$$
\begin{aligned}
& \operatorname{Pr}\left(\max \left\{\epsilon_{1}, \ldots, \epsilon_{j}\right\}<\frac{\epsilon_{0}}{\sqrt{a+1}} \mid \epsilon_{0}, \epsilon_{1}, \ldots, \epsilon_{j}>0\right) \\
& \quad \geq\left(\frac{1}{\sqrt{a+1}}\right)^{j} \cdot \operatorname{Pr}\left(\max \left\{\epsilon_{1}, \ldots, \epsilon_{j}\right\}<\epsilon_{0} \mid \epsilon_{0}, \epsilon_{1}, \ldots, \epsilon_{j}>0\right)=\left(\frac{1}{\sqrt{a+1}}\right)^{j} \frac{1}{j+1} .
\end{aligned}
$$

Summing the contributions of all cells in the partition yields

$$
\begin{aligned}
& r(a, b) \geq 2^{-(b+1)} \cdot\left(\frac{1}{b+1}+\sum_{j=0}^{b}\left(\begin{array}{l}
b \\
j
\end{array}\right)\left(\frac{1}{\sqrt{a+1}}\right)^{j} \frac{1}{j+1}\right) \\
& =\frac{2^{-(b+1)}}{b+1} \cdot\left(1+\sqrt{a+1} \cdot \sum_{j=0}^{b}\left(\begin{array}{c}
b+1 \\
j+1
\end{array}\right)\left(\frac{1}{\sqrt{a+1}}\right)^{j+1}\right)
\end{aligned}
$$


which agrees with $p(a, b)$ by virtue of the binomial theorem.

Lemma A.6: $r(a, b) \geq q(a, b)$.

Proof: Let $\eta_{1}, \ldots, \eta_{a+1}, \epsilon_{0}, \epsilon_{1}, \ldots, \epsilon_{b}$ be i.i.d. random variables that are normally distibuted with mean zero. It is well known that the $\operatorname{sum} \sum_{j} \eta_{j}$ has the same distribution as $\sqrt{a+1} \epsilon_{0}$, so

$$
\begin{aligned}
r(a, b) & =\operatorname{Pr}\left(\frac{\eta_{1}+\ldots+\eta_{a+1}}{a+1}>\max \left\{\epsilon_{1}, \ldots, \epsilon_{j}\right\}\right) \\
& \geq \operatorname{Pr}\left(\min \left\{\eta_{1}, \ldots, \eta_{a+1}\right\}>\max \left\{\epsilon_{1}, \ldots, \epsilon_{j}\right\}\right)=q(a, b) .
\end{aligned}
$$

How accurate are these two lower bounds? Tables 10 and 11 below give the ratios $r(a, b) / p(a, b)$ and $r(a, b) / q(a, b)$ for small $a$ and $b$. Evidently $p$ gives much the better approximation in this range. Also, there is a polynomial function $P_{b}(\cdot)$ such that

$$
\begin{aligned}
p(a, b) & =\frac{2^{-(b+1)}}{b+1} \cdot\left(1+\sqrt{a+1}\left[\left(1+(b+1) \frac{1}{\sqrt{a+1}}+\frac{1}{a+1} P_{b}\left(\frac{1}{\sqrt{a+1}}\right)\right)-1\right]\right) \\
& =\frac{2^{-(b+1)}}{b+1} \cdot\left(b+2+\frac{1}{\sqrt{a+1}} P_{b}\left(\frac{1}{\sqrt{a+1}}\right)\right)
\end{aligned}
$$

so that

$$
\lim _{a \rightarrow \infty} p(a, b)=\frac{2^{-(b+1)}(b+2)}{b+1} \quad \text { while } \quad \lim _{a \rightarrow \infty} q(a, b)=0 .
$$

Therefore $p$ gives a better approximation when $a$ is large in relation to $b$. For fixed $a$, on the other hand, $1 / p(a, b)$ is an exponential function of $b$ while $1 / q(a, b)$ is bounded above by a polynomial function of $b$, so $p(a, b)<q(a, b)$ when $b$ is relatively large. As is illustrated by the lower portion of Table $11, r(a, b) / q(a, b)$ seems to grow at a roughly logarithmic rate as $b$ increases. 


\begin{tabular}{|c|c|c|c|c|c|c|}
\hline \multicolumn{7}{|c|}{ Selected Values of $r(a, b) / p(a, b)$} \\
\hline \multicolumn{7}{|c|}{$\mathbf{~}$} \\
\hline $\mathbf{b}$ & $\mathbf{1}$ & $\mathbf{2}$ & $\mathbf{3}$ & $\mathbf{4}$ & $\mathbf{5}$ & $\mathbf{6}$ \\
\hline $\mathbf{1}$ & 1.079 & 1.118 & 1.143 & 1.160 & 1.174 & 1.184 \\
$\mathbf{2}$ & 1.102 & 1.148 & 1.177 & 1.198 & 1.214 & 1.228 \\
$\mathbf{3}$ & 1.138 & 1.187 & 1.214 & 1.232 & 1.245 & 1.256 \\
$\mathbf{4}$ & 1.193 & 1.251 & 1.275 & 1.287 & 1.295 & 1.300 \\
$\mathbf{5}$ & 1.267 & 1.342 & 1.365 & 1.371 & 1.371 & 1.370 \\
$\mathbf{6}$ & 1.361 & 1.462 & 1.488 & 1.488 & 1.480 & 1.470 \\
$\mathbf{7}$ & 1.474 & 1.615 & 1.647 & 1.643 & 1.625 & 1.605 \\
$\mathbf{8}$ & 1.608 & 1.803 & 1.848 & 1.840 & 1.812 & 1.779 \\
$\mathbf{9}$ & 1.764 & 2.031 & 2.098 & 2.088 & 2.049 & 2.001 \\
$\mathbf{1 0}$ & 1.944 & 2.307 & 2.405 & 2.396 & 2.345 & 2.280 \\
$\mathbf{1 1}$ & 2.152 & 2.639 & 2.782 & 2.777 & 2.712 & 2.627 \\
$\mathbf{1 2}$ & 2.390 & 3.036 & 3.242 & 3.248 & 3.169 & 3.059 \\
$\mathbf{1 3}$ & 2.663 & 3.511 & 3.804 & 3.829 & 3.735 & 3.595 \\
$\mathbf{1 4}$ & 2.975 & 4.080 & 4.492 & 4.546 & 4.438 & 4.263 \\
$\mathbf{1 5}$ & 3.333 & 4.761 & 5.333 & 5.434 & 5.312 & 5.094 \\
\hline
\end{tabular}

Table 10

\begin{tabular}{|c|c|c|c|c|c|}
\hline \multicolumn{6}{|c|}{ Selected Values of $r(a, b) / q(a, b)$} \\
\hline & \multicolumn{5}{|c|}{ a } \\
\hline b & 1 & 2 & 3 & 4 & 5 \\
\hline 1 & 1.500 & 2.000 & 2.500 & 3.000 & 3.500 \\
\hline 2 & 1.825 & 2.902 & 4.231 & 5.810 & 7.639 \\
\hline 3 & 2.061 & 3.706 & 6.057 & 9.239 & 13.375 \\
\hline 4 & 2.246 & 4.427 & 7.911 & 13.132 & 20.588 \\
\hline 5 & 2.397 & 5.077 & 9.754 & 17.372 & 29.132 \\
\hline 6 & 2.523 & 5.669 & 11.567 & 21.866 & 38.858 \\
\hline 7 & 2.632 & 6.211 & 13.341 & 26.548 & 49.626 \\
\hline 8 & 2.727 & 6.711 & 15.069 & 31.365 & 61.309 \\
\hline 9 & 2.812 & 7.175 & 16.749 & 36.277 & 73.794 \\
\hline 10 & 2.888 & 7.609 & 18.382 & 41.254 & 86.979 \\
\hline 100 & 4.457 & 20.040 & 87.916 & 374.104 & 1543.574 \\
\hline 1000 & 5.746 & 34.955 & 214.365 & 1308.393 & 7913.014 \\
\hline 10000 & 6.827 & 50.529 & 382.800 & 2920.290 & 22290.015 \\
\hline 100000 & 7.778 & 66.428 & 585.646 & 5231.428 & 45327.782 \\
\hline
\end{tabular}

Table 11 